\title{
The Impact of High Temperatures on Some Plant Growth Parameters in Some Common Bean Genotypes
}

\author{
Turgay Kabay $^{1 *}$, Aytekin Ekincialp ${ }^{2}$, Suat Sensoy ${ }^{3}$ \\ ${ }^{1}$ Ercis Vocational School, Van Yuzuncu Yil University, 65080 Van, Turkey \\ ${ }^{2}$ Baskale Vocational School, Van Yuzuncu Yil University, 65080 Van, Turkey \\ ${ }^{3}$ Department of Horticulture, Faculty of Agriculture, Van Yuzuncu Yil University 65080 Van, Turkey
}

Received: 8 July 2021

Accepted: 15 October 2021

\begin{abstract}
Common bean is one of the most cultivated vegetables globally and in Turkey. Especially in areas with a temperate climate, the losses in productivity and quality limit production due to high temperatures. Thus, the present study aimed to determine the bean genotypes tolerant to high temperature stress and to reduce the above-mentioned yield and quality losses to benefit the researchers and producers. In the present study, 46 bean genotypes and 2 bean cultivars, determined to be tolerant and sensitive to high temperatures in previous studies, were used. Variations in 0-5 visual scale value, plant fresh and dry weight, root fresh and dry weight, stem diameter, plant height, leaf number and area, relative leaf water content, membrane damage index and ion ( $\mathrm{K}$ and $\mathrm{Ca}$ ) content were determined in bean plants. Bean seeds were sown in 2-liter pots containing 2:1 peat + perlite mixture, 3 plants per pot based on the randomized blocks experimental design. From the seed sowing until the first real leaf appearance, the pots were irrigated with tap water and then, the pots were irrigated with Hoagland nutrient solution. The control plants were grown in a polyethylene greenhouse and the plants subjected to high temperature were grown in an extra polyethylene covered high tunnel in a polyethylene greenhouse to create high temperature stress. There was a daytime temperature difference of $6^{\circ} \mathrm{C}$ (optimum: $32.17^{\circ} \mathrm{C}$ for high temperature application; optimum: $26.17^{\circ} \mathrm{C}$ for control application) between the control and high temperature applications. The analyzes conducted on the plants revealed that the genotypes V21, V28, $\mathrm{V} 32$, V82, T65, and V-a1 and cv. Yakutiye were tolerant to high temperature stress, while the genotypes V88, V89, T7, and T72 and cv. Zulbiye were the sensitive ones.
\end{abstract}

Keywords: high temperature, ion, plant growth, Phaseolus vulgaris L.

*e-mail: turgaykabay@gmail.com 


\section{Introduction}

Increase in temperatures during bean cultivation period leads to necrotic injuries and changes in cellular functions, especially in the stem and the leaves. [13]. Nutrient intake of the plants depends on root morphology, soil properties, drought and plant species. Drought, extreme temperatures and mineral nutrition are closely related. Higher temperatures affect nutrient intake negatively and reduce mineral intake $[4,5]$.

Two of the basic elements required for growth and development are $\mathrm{K}$ and $\mathrm{Ca}$ ions. Potassium $(\mathrm{K})$ is an essential nutrient for plants and is the most prevalent cation in plants. If the $\mathrm{K}$ and $\mathrm{Ca}$ contents of a plant are sufficient, transpiration would be under control and the opening and closure of stomata would exhibit a linear correlation with the $\mathrm{K}$ content in specialized stoma cells.

In a study where mung beans were exposed to three different temperatures, and it was determined that in high temperatures, the mean shoot biomass (including root) in plants decreased by $9 \%$ and $41 \%$, and there were significant differences between the genotypes based on root weight; however, there was a positive correlation between root and shoot weights [6]. It was reported that high temperatures in greenhouse conditions leads to decreases in growth, yield and relative water content in hyacinth bean [7]. In a study where French bean genotypes were exposed to different high temperature regimes; it was found that the plant numbers decreased across the genotypes [8].

It was reported that corn grain yield in high temperatures was reduced by $80-90 \%$ when compared to normal temperatures [9]. It was reported in a study conducted with two soybean genotypes (JS 97-52 and EC 538828) under high temperature and arid conditions that there were decreases in several growth traits [10]. In a study where peanut genotypes were analyzed based on pod yield and physiological parameters under heat stress and in environments without stress, it was reported that pod yield, 100-seed weight and pod growth rate could be used as the criteria in selection of heat tolerant genotypes and based on stress tolerance index and pod yield performance; and some genotypes were determined as heat-tolerant ones [11]. In a study investigating the effects of high temperature stress on yield in pepper genotypes, it was reported that high temperatures would negatively affect pepper growth and yield [12].

High temperature stress also leads to drought damages since the water intake of the plants is adversely affected. In a study aiming to determine the tolerance and sensitivity of 46 bean genotypes and 2 bean cultivars to drought stress, it was determined that the genotypes V7, V15, V33, V82, V89, and V-A1, and cv. Yakutiye were tolerant to drought and the genotypes V21, V62, V69, V71, V86, V95, and T7 and cv. Zulbiye were sensitive to drought [13]. It was reported that leaf area and number, plant height and weight, stem diameter and relative leaf water content, and $\mathrm{K}$ and $\mathrm{Ca}$ content decreased in crop species under drought stress, while the membrane damage index increased [13-16]. It was indicated that the plant weight, leaf number, stem diameter and relative leaf water content decreased and membrane damage index increased in tomato plants exposed to drought stress [17, 18]. In tomato cultivars exposed to drought stress, the fresh and dry weight, leaf area and relative leaf water content significantly decreased in all cultivars when compared to the control group, while in bean plants, water and high temperature stress adversely affected the growth of sensitive bean cultivars [19].

In Turkey, which is the genetic center for various vegetables, the production and consumption of beans is prevalent in every single region for several years. Thus, there are several bean genotypes in each region in Turkey. The objective of the present study was to determine high temperature stress tolerant genotypes among them, would provide benefits for both the producers and researchers.

\section{Material and Methods}

In the present study conducted to determine genotypes that are high temperature stress tolerant and sensitive, 46 bean genotypes and two cultivars (cv. Yakutiye determined as abiotic stress tolerant and cv. Zulbiye determined as abiotic stress sensitive in previous studies) were investigated and the study was constructed with a randomized complete block experimental design with 4 replications. The seeds were sown in 2-liter plastic pots containing 2:1 peat: perlite mixture and as 3 seedlings per pot.

The seeds were irrigated with controlled tap water. The plants were irrigated with the standard nutrient solution until harvest after the formation of first true leaves. The irrigation was based on "drained solution/ applied solution" [20].

The control plants were grown in a covered greenhouse, while the plants that were exposed to high temperature stress were grown in a high tunnel with a second polyethylene cover in the same greenhouse. There was a daytime temperature difference of $6^{\circ} \mathrm{C}$ (optimum: $32.17^{\circ} \mathrm{C}$ for high temperature application; optimum: $26.17^{\circ} \mathrm{C}$ for control application) between the control and high temperature applications. The nighttime temperature difference between the high temperature stress application and the control application was $2.91^{\circ} \mathrm{C}$ (optimum: $14.98^{\circ} \mathrm{C}$ for high temperature application; optimum: $12.07^{\circ} \mathrm{C}$ for control application). Analyzes were conducted on the samples obtained from the common bean plants after termination of the study on the $37^{\text {th }}$ day.

\section{Evaluation with a $0-5$ Scale}

Bean genotypes were rated for their high temperature tolerance on a $0-5$ scale compared to check 
plants: $0=$ healthy plants with no visible symptoms of high temperature stress; $1=$ decrease in development; $2=$ slight wilting in older leaves; $3=$ moderate curling and wilting in upper leaves; $4=$ leaves yellow-brown with severe wilting; and $5=$ Wilted plants and dried lower leaves [20-22].

\section{Determination of Fresh and Dry Weights}

All plants were weighed on a precision scale ( $\pm 0.1 \mathrm{~g})$. Then, the samples were open air dried one day, and oven dried for 48 hours at $65^{\circ} \mathrm{C}$ till reach a constant weight, and the dried samples were weighed on a precision scale $( \pm 0.1 \mathrm{~g})$.

\section{Determination of the Plant Height and Diameter}

The stem lengths in bean plants were measured with a ruler $( \pm 0.1 \mathrm{~cm})$, and their stem diameters were measured with a digital display caliper $( \pm 0.1 \mathrm{~mm})$.

\section{Determination of the Number of Leaves and Leaf Area}

At the end of the high temperature experiment, the number of leaves was counted and the leaf areas were determined as $\mathrm{cm}^{2}$ with a planimeter in all bean genotypes.

\section{Determination of the Leaf Relative Water Content (LRWC)}

At the end of the high temperature experiment, fresh leaf samples were sampled, weighted (FW) and kept in distilled water for four hours to calculate their turgor weights (TW). Then, the samples were kept in an oven $\left(65^{\circ} \mathrm{C}\right)$ for 48 hours and weighted (DW). The below formula was used in order to calculate the relative water content of the bean genotypes [20, 23, 24].

$$
\text { LRWC }=\frac{(\text { FW-DW })}{(\text { TW-DW })} \times 100
$$

\section{Determination of Relative Growth Rate ( $\mathrm{g}$ fresh weight day $^{-1}$ )}

Before and after high temperature stress applications, the plants were weighed and the difference between the two measurements was divided by the number of days [20].

\section{Determination of the Membrane Damages in the Leaf Cells}

Membrane Damage Index (MDI) in bean leaves was calculated by measuring the electrolyte out of the cell. The $17 \mathrm{~mm}$ diameter discs taken from the bottom 3 leaves were incubated for 5 hours in $10 \mathrm{ml}$ distilled water, and their EC values were measured. The same disc samples were kept at $100 \mathrm{C}^{\circ}$ for 10 minutes, and their EC values were measured again. Membrane Damage Index (MDI) was calculated by the following formula $[20,25,26]$.

$$
\mathrm{MDI}=\frac{\mathrm{Lt}-\mathrm{Lc}}{1-\mathrm{Lc}} \times 100
$$

Lt: The first EC value of high temperature stressed leaf disk samples / The second EC value of high temperature stressed leaf disc samples kept at $100^{\circ} \mathrm{C}$ for 10 minutes. Lc: The first EC value of control leaf disk samples / The second EC value of control disc samples kept at $100^{\circ} \mathrm{C}$ for 10 minutes .

\section{Mineral Element Analysis}

At the end of the high temperature experiment, the shoot and root samples from high temperature stressed and control plants were dried in an oven at $65^{\circ} \mathrm{C}$ until reach a constant weight. Then the dry samples $(200 \mathrm{mg})$ were ground, pre-lit by ethyl alcohol, and lit till ash formation at $550^{\circ} \mathrm{C}$. The ash samples were dissolved with a $3.3 \% \mathrm{HCl}$ solution, filtered with a blue-band filter paper, and $\mathrm{Na}, \mathrm{K}$, and $\mathrm{Ca}$ was determined in an atomic absorption device (Thermo trade brand serial no: ice3000 series aa spectrometer) [20, 22, 27].

\section{Statistical Analysis}

Analysis of Variances based on general linear models [28] carried out by SAS 9.4.1 statistical program was used. Duncan's multiple comparison test was used to measure the statistical differences between genotype.

\section{Results and Discussion}

High temperatures during bean cultivation reduce the plant yield and quality. Thus, the availability of genotypes and cultivars that are tolerant to high temperatures would have a positive impact on bean production, helping to relieve related problems of producers and researchers. In the present study, it was found that the genotypes V21, V28, V32, V82, T65, $\mathrm{V}$-al and cv. Yakutiye on the 0-5 visual scale were statistically tolerant and the genotypes V88, V89, T7, T72 and cv. Zulbiye were statistically sensitive to high temperatures (Table 1). The genotype V21 was the least affected genotype based on the growth rate under high temperature stress, while the growth rate was $0.506 \%$ in cv. Yakutiye. It was determined that the lowest growth rates were observed in the genotypes V89 and $\mathrm{T} 72$, while the growth rate was $0.124 \%$ in $\mathrm{cv}$. Zulbiye (Table 1). The highest relative leaf water content under high temperature stress was observed in the genotype T65 with $80.678 \%$ and the lowest relative leaf water content was observed in the genotype T121 with 
Table 1. 0-5 scale value, relative plant growth rate (RPGR) (\%) and leaf relative water content (LRWC) (\%) of the common bean genotypes in the high temperature stress study.

\begin{tabular}{|c|c|c|c|c|c|}
\hline $\begin{array}{c}\text { Bean } \\
\text { Genotype }\end{array}$ & $\begin{array}{l}0-5 \text { scale in high } \\
\text { temperature stress }\end{array}$ & $\begin{array}{l}\text { RPGR in control } \\
(\%)\end{array}$ & $\begin{array}{c}\text { RPGR in high } \\
\text { temperature stress }(\%)\end{array}$ & $\begin{array}{c}\text { LRWC in control } \\
(\%)\end{array}$ & $\begin{array}{c}\text { LRWC in high } \\
\text { temperature stress }(\%)\end{array}$ \\
\hline V5 & $2.500 \mathrm{gh}^{*}$ & $0.279 \mathrm{v}^{*}$ & 0.191 w-y* & 73.804 m-s* & $64.237 \mathrm{~h}-\mathrm{j} *$ \\
\hline V6 & 1.750 hij & 0.684 e-i & $0.475 \mathrm{a}-\mathrm{c}$ & 80.879 l-h & $71.941 \mathrm{~d}-\mathrm{f}$ \\
\hline V7 & $4.250 \mathrm{abc}$ & $0.659 \mathrm{i}-\mathrm{k}$ & $0.215 \mathrm{t}-\mathrm{w}$ & 60.857 xyz & $45.175 \mathrm{tu}$ \\
\hline V10 & $1.500 \mathrm{ij}$ & $0.514 \mathrm{~s}-\mathrm{u}$ & $0.331 \mathrm{kl}$ & 65.352 vwx & $58.731 \mathrm{k}-\mathrm{o}$ \\
\hline V12 & $2.500 \mathrm{gh}$ & $0.686 \mathrm{e}-\mathrm{h}$ & $0.291 \mathrm{~m}-\mathrm{o}$ & $59.176 \mathrm{z}$ & $47.996 \mathrm{st}$ \\
\hline V14 & $1.500 \mathrm{ij}$ & $0.606 \mathrm{~m}-\mathrm{o}$ & $0.406 \mathrm{f}-\mathrm{h}$ & $78.731 \mathrm{e}-1$ & $71.500 \mathrm{~d}-\mathrm{f}$ \\
\hline V15 & $1.500 \mathrm{ij}$ & $0.485 \mathrm{u}$ & $0.335 \mathrm{kl}$ & 74.627 k-r & $69.357 \mathrm{e}-\mathrm{g}$ \\
\hline V21 & $1.250 \mathrm{j}$ & $0.736 \mathrm{~b}$ & $0.509 \mathrm{a}$ & $86.454 \mathrm{ab}$ & $78.388 \mathrm{ab}$ \\
\hline V28 & $1.250 \mathrm{j}$ & $0.537 \mathrm{r}-\mathrm{t}$ & $0.359 \mathrm{i}-\mathrm{k}$ & $63.845 \mathrm{v}-\mathrm{y}$ & $58.728 \mathrm{k}-\mathrm{o}$ \\
\hline V29 & $1.500 \mathrm{ij}$ & $0.553 \mathrm{p}-\mathrm{s}$ & $0.375 \mathrm{ij}$ & $58.557 \mathrm{z}$ & $52.579 \mathrm{qr}$ \\
\hline V30 & $4.250 \mathrm{abc}$ & $0.499 \mathrm{tu}$ & $0.210 u-x$ & $60.322 \mathrm{yz}$ & $43.344 \mathrm{uv}$ \\
\hline V32 & $1.250 \mathrm{j}$ & $0.643 \mathrm{i}-\mathrm{m}$ & $0.442 \mathrm{c}-\mathrm{f}$ & $84.180 \mathrm{a}-\mathrm{d}$ & $76.775 \mathrm{bc}$ \\
\hline V33 & 1.750 hij & $0.590 \mathrm{n}-\mathrm{p}$ & $0.3021-n$ & $78.881 \mathrm{e}-1$ & $69.293 \mathrm{e}-\mathrm{g}$ \\
\hline V36 & $2.750 \mathrm{efg}$ & $0.612 \mathrm{~m}-\mathrm{o}$ & $0.206 \mathrm{v}-\mathrm{x}$ & $82.343 \mathrm{~b}-\mathrm{f}$ & 66.209 h-i \\
\hline V40 & $1.500 \mathrm{ij}$ & $0.519 \mathrm{~s}-\mathrm{u}$ & $0.322 \mathrm{k}-\mathrm{m}$ & 60.962 xyz & $56.320 \mathrm{~m}-\mathrm{q}$ \\
\hline V41 & $4.250 \mathrm{abc}$ & $0.538 \mathrm{r}-\mathrm{t}$ & $0.217 \mathrm{~s}-\mathrm{w}$ & $69.978 \mathrm{stu}$ & $53.133 \mathrm{p}-\mathrm{r}$ \\
\hline V43 & $4.500 \mathrm{ab}$ & $0.584 \mathrm{o}-\mathrm{q}$ & $0.155 \mathrm{yz}$ & 68.047 tuv & $46.676 \mathrm{tu}$ \\
\hline V47 & $3.500 \mathrm{cde}$ & $0.638 \mathrm{j}-\mathrm{m}$ & $0.217 \mathrm{~s}-\mathrm{u}$ & $58.996 \mathrm{z}$ & $44.254 \mathrm{t}-\mathrm{v}$ \\
\hline V49 & $3.500 \mathrm{cde}$ & $0.717 \mathrm{~b}-\mathrm{d}$ & $0.288 \mathrm{~m}-\mathrm{p}$ & 65.424 vwx & $47.804 \mathrm{st}$ \\
\hline V57 & $4.250 \mathrm{abc}$ & $0.672 \mathrm{~d}-\mathrm{k}$ & $0.279 \mathrm{n}-\mathrm{p}$ & 76.799 g-n & $55.474 \mathrm{n}-\mathrm{q}$ \\
\hline V62 & $3.750 \mathrm{bd}$ & $0.695 \mathrm{~b}-\mathrm{g}$ & $0.3031-n$ & $75.749 \mathrm{i}-\mathrm{q}$ & $57.1781-\mathrm{p}$ \\
\hline V63 & $4.250 \mathrm{abc}$ & $0.6171-\mathrm{o}$ & $0.252 \mathrm{o}-\mathrm{t}$ & $79.792 \mathrm{~d}-\mathrm{j}$ & $58.656 \mathrm{k}-\mathrm{o}$ \\
\hline V64 & $1.500 \mathrm{ij}$ & $0.663 \mathrm{f}-\mathrm{k}$ & $0.467 \mathrm{bc}$ & $83.227 \mathrm{a}-\mathrm{e}$ & $75.440 \mathrm{~b}-\mathrm{d}$ \\
\hline V66 & $1.500 \mathrm{ij}$ & $0.658 \mathrm{~g}-\mathrm{k}$ & $0.447 \mathrm{c}-\mathrm{e}$ & $66.521 \mathrm{uvw}$ & $60.964 \mathrm{j}-1$ \\
\hline V69 & 1.750 hij & $0.791 \mathrm{a}$ & $0.448 \mathrm{c}-\mathrm{e}$ & $85.224 \mathrm{abc}$ & $76.750 \mathrm{abc}$ \\
\hline V71 & $2.750 \mathrm{efg}$ & 0.575 o-r & $0.215 \mathrm{t}-\mathrm{w}$ & $61.040 \mathrm{xyz}$ & $47.449 \mathrm{st}$ \\
\hline V73 & $2.750 \mathrm{efg}$ & $0.801 \mathrm{a}$ & $0.258 \mathrm{o}-\mathrm{s}$ & $80.196 \mathrm{~d}-\mathrm{i}$ & $59.881 \mathrm{k}-\mathrm{m}$ \\
\hline V77 & $2.500 \mathrm{gh}$ & $0.657 \mathrm{~g}-1$ & $0.248 \mathrm{p}-\mathrm{u}$ & 79.037 e-k & $60.431 \mathrm{j}-\mathrm{m}$ \\
\hline V79 & $3.250 \mathrm{def}$ & $0.706 \mathrm{~b}-\mathrm{f}$ & $0.251 \mathrm{o}-\mathrm{u}$ & $81.109 \mathrm{c}-\mathrm{g}$ & $59.039 \mathrm{k}-\mathrm{n}$ \\
\hline V80 & $3.250 \mathrm{def}$ & $0.712 \mathrm{~b}-\mathrm{d}$ & $0.252 \mathrm{o}-\mathrm{t}$ & $66.385 \mathrm{uvw}$ & $47.572 \mathrm{st}$ \\
\hline V82 & $1.250 \mathrm{j}$ & $0.598 \mathrm{~m}-\mathrm{o}$ & $0.415 \mathrm{e}-\mathrm{g}$ & $75.209 \mathrm{jr}$ & $69.004 \mathrm{fg}$ \\
\hline V86 & 1.750 hij & $0.554 \mathrm{p}-\mathrm{s}$ & 0.386 g-i & $74.1121-\mathrm{s}$ & $66.937 \mathrm{hg}$ \\
\hline V88 & $4.750 \mathrm{a}$ & $0.632 \mathrm{k}-\mathrm{n}$ & $0.174 x-z$ & $75.096 \mathrm{jr}$ & $50.975 \mathrm{rs}$ \\
\hline V89 & $4.750 \mathrm{a}$ & $0.544 \mathrm{q}-\mathrm{s}$ & $0.128 \mathrm{z}$ & 61.724 xyz & $42.704 \mathrm{uv}$ \\
\hline V90 & $1.750 \mathrm{~h}-\mathrm{j}$ & $0.599 \mathrm{~m}-\mathrm{o}$ & $0.349 \mathrm{i}-\mathrm{k}$ & $72.926 \mathrm{n}-\mathrm{s}$ & 60.054 k-m \\
\hline V95 & $1.750 \mathrm{~h}-\mathrm{j}$ & 0.669 e-k & $0.339 \mathrm{j}-1$ & 76.203 h-p & $60.508 \mathrm{j}-\mathrm{m}$ \\
\hline V99 & $1.500 \mathrm{ij}$ & $0.719 \mathrm{bc}$ & $0.459 \mathrm{~b}-\mathrm{d}$ & $83.268 \mathrm{a}-\mathrm{e}$ & $73.284 \mathrm{c}-\mathrm{e}$ \\
\hline $\mathrm{T} 7$ & $4.750 \mathrm{a}$ & $0.608 \mathrm{~m}-\mathrm{o}$ & $0.148 \mathrm{yz}$ & $71.829 \mathrm{o}-\mathrm{t}$ & $47.555 \mathrm{st}$ \\
\hline
\end{tabular}


Table 1. Continued.

\begin{tabular}{|c|c|c|c|c|c|}
\hline T32 & $3.500 \mathrm{c}-\mathrm{e}$ & $0.717 \mathrm{~b}-\mathrm{d}$ & $0.259 \mathrm{o}-\mathrm{r}$ & $77.785 \mathrm{~g}-\mathrm{m}$ & $57.0091-\mathrm{p}$ \\
\hline T37 & $2.500 \mathrm{gh}$ & $0.593 \mathrm{o}-\mathrm{p}$ & $0.272 \mathrm{n}-\mathrm{q}$ & $76.599 \mathrm{~g}-\mathrm{n}$ & $62.509 \mathrm{i}-\mathrm{k}$ \\
\hline T65 & $1.250 \mathrm{j}$ & $0.639 \mathrm{i}-\mathrm{m}$ & $0.422 \mathrm{~d}-\mathrm{g}$ & $87.330 \mathrm{a}$ & $80.678 \mathrm{a}$ \\
\hline T72 & $4.750 \mathrm{a}$ & $0.691 \mathrm{~b}-\mathrm{g}$ & $0.134 \mathrm{z}$ & $62.667 \mathrm{w}-\mathrm{z}$ & $41.508 \mathrm{v}$ \\
\hline T121 & $3.750 \mathrm{bcd}$ & $0.705 \mathrm{~b}-\mathrm{f}$ & $0.228 \mathrm{r}-\mathrm{w}$ & $71.397 \mathrm{q}-\mathrm{t}$ & $54.817 \mathrm{o}-\mathrm{r}$ \\
\hline V-e1 & $4.250 \mathrm{abc}$ & $0.669 \mathrm{e}-\mathrm{k}$ & $0.256 \mathrm{o}-\mathrm{t}$ & $74.755 \mathrm{k}-\mathrm{r}$ & $54.766 \mathrm{o}-\mathrm{r}$ \\
\hline V-a1 & $1.250 \mathrm{j}$ & $0.681 \mathrm{c}-\mathrm{j}$ & $0.487 \mathrm{ab}$ & $76.399 \mathrm{~g}-\mathrm{o}$ & $71.202 \mathrm{ef}$ \\
\hline V-g1 & $3.250 \mathrm{def}$ & $0.680 \mathrm{c}-\mathrm{j}$ & $0.235 \mathrm{r}-\mathrm{v}$ & $70.779 \mathrm{r}-\mathrm{u}$ & $52.503 \mathrm{qr}$ \\
\hline Zulbiye & $4.750 \mathrm{a}$ & $0.633 \mathrm{k}-\mathrm{n}$ & $0.124 \mathrm{z}$ & $71.544 \mathrm{p}-\mathrm{t}$ & $47.478 \mathrm{st}$ \\
\hline Yakutiye & $1.250 \mathrm{j}$ & $0.705 \mathrm{~b}-\mathrm{f}$ & $0.506 \mathrm{a}$ & $77.816 \mathrm{f}-\mathrm{m}$ & $72.467 \mathrm{de}$ \\
\hline
\end{tabular}

* There is a significant difference $(\mathrm{p}<0.05)$ among the different letters in each column.

41.508\% (Table 1). The membrane damage was highest in the genotypes T7, V89 and V88 and cv. Zulbiye, while it was the lowest in the genotypes V-a1, V14, V21, V28, V29, V32, and V99 and cv. Yakutiye. Statistically, plant height loss was the least in the genotype V-al under high temperature stress, while the genotype T7 exhibited the highest plant height loss. It can be observed in Table 2 that the leaf number and area loss under high temperature stress were the lowest in the genotypes $\mathrm{V}$-al and V15 and the maximum leaf area loss was observed in the genotype T7. It was reported that high temperatures in greenhouse conditions lead to losses in growth, yield and relative leaf water content in hyacinth bean (Lablab purpureus. L) plants [7]. It was indicated that when the French bean genotypes were exposed to high temperature stress, the decrease

Table 2. Membrane damage Index (MDI) (\%), Shoot height (cm), Leaf area $\left(\mathrm{cm}^{2}\right)$ and Leaf number of the bean genotypes in the high temperature stress study.

\begin{tabular}{|c|c|c|c|c|c|c|c|}
\hline $\begin{array}{c}\text { Bean } \\
\text { Genotype }\end{array}$ & $\begin{array}{l}\text { MDI in high } \\
\text { temperature } \\
\text { stress }(\%)\end{array}$ & $\begin{array}{l}\text { Shoot height } \\
\text { in control } \\
(\mathrm{cm})\end{array}$ & $\begin{array}{l}\text { Shoot height in } \\
\text { high temperature } \\
\text { stress }(\mathrm{cm})\end{array}$ & $\begin{array}{l}\text { Leaf area in } \\
\text { control }\left(\mathrm{cm}^{2}\right)\end{array}$ & $\begin{array}{c}\text { Leaf area in high } \\
\text { temperature } \\
\text { stress }\left(\mathrm{cm}^{2}\right) \\
\end{array}$ & $\begin{array}{l}\text { Leaf number } \\
\text { in control }\end{array}$ & $\begin{array}{l}\text { Leaf number in } \\
\text { high temperature } \\
\text { stress }\end{array}$ \\
\hline V5 & $25.517 \mathrm{k}-\mathrm{m}^{*}$ & $53.225 \mathrm{j}-\mathrm{n}^{*}$ & 40.400 g-k* & $30.346 \mathrm{a}-\mathrm{h} *$ & 17.769 f-o* & $14.500 \mathrm{a}^{*}$ & 11.750 a-d* \\
\hline V6 & $22.567 \mathrm{~lm}$ & 66,025 e-g & $52.775 \mathrm{f}$ & 29.522 a-h & $22.283 \mathrm{a}-\mathrm{i}$ & $12.500 \mathrm{a}-\mathrm{g}$ & $9.750 \mathrm{~d}-\mathrm{h}$ \\
\hline V7 & $44.500 \mathrm{c}-\mathrm{h}$ & 46.875 n-s & $38.975 \mathrm{~g}-\mathrm{k}$ & 29.761 a-h & $11.311 \mathrm{o}-\mathrm{r}$ & $11.750 \mathrm{~b}-\mathrm{g}$ & $6.5001-q$ \\
\hline V10 & $20.764 \mathrm{~lm}$ & $36.475 \mathrm{uv}$ & $29.900 \mathrm{~m}-\mathrm{o}$ & $26.523 \mathrm{c}-\mathrm{h}$ & $19.396 \mathrm{~b}-1$ & $12.250 \mathrm{a}-\mathrm{g}$ & $10.000 \mathrm{c}-\mathrm{h}$ \\
\hline V12 & 27.645 i-m & $39.050 \mathrm{~s}-\mathrm{v}$ & $31.550 \mathrm{mn}$ & 36.445 a-d & $25.586 \mathrm{a}-\mathrm{c}$ & $14.250 \mathrm{ab}$ & $10.000 \mathrm{c}-\mathrm{h}$ \\
\hline V14 & $19.873 \mathrm{~m}$ & $46.750 \mathrm{n}-\mathrm{s}$ & $29.950 \mathrm{~m}-\mathrm{o}$ & 25.900 e-h & 18.337 e-n & $12.250 \mathrm{a}-\mathrm{g}$ & $11.000 \mathrm{~b}-\mathrm{e}$ \\
\hline V15 & $20.240 \mathrm{~lm}$ & $91.925 \mathrm{a}$ & $82.725 \mathrm{a}$ & $36.253 \mathrm{a}-\mathrm{e}$ & $27.506 \mathrm{a}$ & $12.250 \mathrm{a}-\mathrm{g}$ & $9.750 \mathrm{~d}-\mathrm{h}$ \\
\hline V21 & $18.380 \mathrm{~m}$ & $63.375 \mathrm{~g}-\mathrm{i}$ & $54.625 \mathrm{f}$ & $24.822 \mathrm{f}-\mathrm{h}$ & 17.670 i-o & $12.500 \mathrm{a}-\mathrm{g}$ & $10.500 \mathrm{c}-\mathrm{f}$ \\
\hline V28 & $19.141 \mathrm{~m}$ & $60.425 \mathrm{~g}-\mathrm{j}$ & $52.375 \mathrm{f}$ & $31.159 \mathrm{a}-\mathrm{h}$ & $22.417 \mathrm{a}-\mathrm{i}$ & $14.250 \mathrm{ab}$ & $12.500 \mathrm{ab}$ \\
\hline V29 & $18.384 \mathrm{~m}$ & $78.775 \mathrm{~cd}$ & $70.500 \mathrm{~b}$ & $26.922 \mathrm{c}-\mathrm{h}$ & $16.160 \mathrm{i}-\mathrm{q}$ & $12.000 \mathrm{a}-\mathrm{g}$ & $10.000 \mathrm{c}-\mathrm{h}$ \\
\hline V30 & $45.650 \mathrm{c}-\mathrm{f}$ & $64.375 \mathrm{f}-\mathrm{i}$ & $42.125 \mathrm{~g}-\mathrm{i}$ & $24.772 \mathrm{f}-\mathrm{h}$ & 10.975 o-r & $10.750 \mathrm{afg}$ & $6.750 \mathrm{k}-\mathrm{p}$ \\
\hline V32 & $18.456 \mathrm{~m}$ & 59.700 g-k & $52.000 \mathrm{f}$ & $30.771 \mathrm{a}-\mathrm{h}$ & 24.826 a-e & $13.000 \mathrm{a}-\mathrm{f}$ & $11.750 \mathrm{a}-\mathrm{d}$ \\
\hline V33 & $22.4261-\mathrm{m}$ & $50.750 \mathrm{l}-\mathrm{q}$ & $43.125 \mathrm{gh}$ & $37.594 \mathrm{ab}$ & $26.088 \mathrm{ab}$ & $11.750 \mathrm{~b}-\mathrm{g}$ & $10.250 \mathrm{c}-\mathrm{f}$ \\
\hline V36 & $29.176 \mathrm{i}-\mathrm{m}$ & $44.125 \mathrm{o}-\mathrm{t}$ & $29.950 \mathrm{~m}-\mathrm{o}$ & $36.835 \mathrm{a}-\mathrm{c}$ & $20.518 \mathrm{~b}-\mathrm{j}$ & 13.250 a-e & $8.750 \mathrm{f}-\mathrm{k}$ \\
\hline V40 & $20.721 \mathrm{~lm}$ & $51.650 \mathrm{k}-\mathrm{p}$ & $44.500 \mathrm{gh}$ & 29.030 a-h & 20.130 b-k & $10.500 \mathrm{fg}$ & 9.000 e-j \\
\hline V41 & $46.190 \mathrm{~b}-\mathrm{f}$ & $56.200 \mathrm{i}-\mathrm{m}$ & $36.525 \mathrm{i}-1$ & $29.521 \mathrm{a}-\mathrm{h}$ & $9.210 \mathrm{qr}$ & $11.250 \mathrm{c}-\mathrm{g}$ & 8.000 h-n \\
\hline V43 & $50.154 \mathrm{a}-\mathrm{d}$ & $51.500 \mathrm{k}-\mathrm{p}$ & $25.900 n-p$ & 32.396 a-h & 14.187 j-r & $11.500 \mathrm{c}-\mathrm{g}$ & $5.500 \mathrm{opq}$ \\
\hline
\end{tabular}


Table 2. Continued.

\begin{tabular}{|c|c|c|c|c|c|c|c|}
\hline V47 & $35.053 \mathrm{f}-\mathrm{k}$ & $67.500 \mathrm{e}-\mathrm{g}$ & $45.450 \mathrm{~g}$ & 26.319 d-h & 13.590 k-r & $11.750 \mathrm{~b}-\mathrm{g}$ & $7.500 \mathrm{i}-\mathrm{o}$ \\
\hline V49 & 37.700 e-i & $87.250 \mathrm{ab}$ & $60.700 \mathrm{de}$ & $28.226 \mathrm{a}-\mathrm{h}$ & 13.828 j-r & $12.500 \mathrm{a}-\mathrm{g}$ & 8.000 h-m \\
\hline V57 & $43.695 \mathrm{~d}-\mathrm{h}$ & $53.775 \mathrm{j}-\mathrm{n}$ & $34.025 \mathrm{k}-\mathrm{m}$ & $27.456 \mathrm{~b}-\mathrm{h}$ & $11.822 \mathrm{~m}-\mathrm{r}$ & $13.000 \mathrm{a}-\mathrm{f}$ & $8.750 \mathrm{f}-\mathrm{k}$ \\
\hline V62 & 38.599 e-i & $33.125 \mathrm{v}$ & $20.550 p$ & $25.107 \mathrm{f}-\mathrm{h}$ & 11.385 o-r & $12.000 \mathrm{a}-\mathrm{g}$ & $7.500 \mathrm{i}-\mathrm{o}$ \\
\hline V63 & $46.697 \mathrm{~b}-\mathrm{e}$ & $63.850 \mathrm{f}-\mathrm{i}$ & $42.150 \mathrm{~g}-\mathrm{i}$ & 29.700 a-h & $11.495 \mathrm{n}-\mathrm{r}$ & $12.250 \mathrm{a}-\mathrm{g}$ & $7.250 \mathrm{j}-\mathrm{o}$ \\
\hline V64 & $20.139 \mathrm{~lm}$ & $78.975 \mathrm{~cd}$ & $68.200 \mathrm{bc}$ & $30.122 \mathrm{a}-\mathrm{h}$ & $20.541 \mathrm{~b}-\mathrm{j}$ & $11.500 \mathrm{c}-\mathrm{g}$ & $9.500 \mathrm{e}-\mathrm{i}$ \\
\hline V66 & $19.506 \mathrm{~lm}$ & $72.875 \mathrm{de}$ & $63.100 \mathrm{~cd}$ & $37.917 \mathrm{a}$ & $24.384 \mathrm{a}-\mathrm{g}$ & $11.500 \mathrm{c}-\mathrm{g}$ & $9.500 \mathrm{e}-\mathrm{i}$ \\
\hline V69 & $20.7801-\mathrm{m}$ & $62.958 \mathrm{~g}-\mathrm{i}$ & $53.675 \mathrm{f}$ & $26.349 \mathrm{~d}-\mathrm{h}$ & $14.245 \mathrm{j}-\mathrm{r}$ & $12.250 \mathrm{a}-\mathrm{g}$ & $10.500 \mathrm{c}-\mathrm{f}$ \\
\hline V71 & 34.477 g-k & $42.700 \mathrm{q}-\mathrm{t}$ & $26.025 \mathrm{n}-\mathrm{p}$ & $22.827 \mathrm{~h}$ & $9.070 \mathrm{r}$ & $11.500 \mathrm{c}-\mathrm{g}$ & $8.500 \mathrm{f}-1$ \\
\hline V73 & 33.964 h-k & $84.325 \mathrm{cb}$ & 56.925 ef & $31.318 \mathrm{a}-\mathrm{h}$ & $17.293 \mathrm{i}--\mathrm{o}$ & $12.750 \mathrm{a}-\mathrm{g}$ & $7.500 \mathrm{i}-\mathrm{o}$ \\
\hline V77 & 31.873 i-1 & $60.500 \mathrm{~g}-\mathrm{j}$ & $36.250 \mathrm{i}-\mathrm{m}$ & $22.747 \mathrm{~h}$ & 10.947 o-r & $12.000 \mathrm{a}-\mathrm{g}$ & $7.500 \mathrm{i}-\mathrm{o}$ \\
\hline V79 & 35.754 e-k & $53.325 \mathrm{j}-\mathrm{n}$ & $34,225 \mathrm{j}-\mathrm{m}$ & $27.568 \mathrm{a}-\mathrm{h}$ & $13.492 \mathrm{k}-\mathrm{r}$ & $11.250 \mathrm{c}-\mathrm{g}$ & $7.250 \mathrm{j}-\mathrm{o}$ \\
\hline V80 & $37.058 \mathrm{e}-\mathrm{j}$ & 47.625 n-r & $32.1751-n$ & $32.782 \mathrm{a}-\mathrm{h}$ & $18.936 \mathrm{c}-1$ & $13.000 \mathrm{a}-\mathrm{f}$ & $8.250 \mathrm{~g}-\mathrm{m}$ \\
\hline V82 & $18.645 \mathrm{~m}$ & $45.400 \mathrm{n}-\mathrm{t}$ & 40.375 g-k & 29.665 a-h & 23.922 a--h & $11.250 \mathrm{c}-\mathrm{g}$ & $10.250 \mathrm{c}-\mathrm{g}$ \\
\hline V86 & $22.092 \mathrm{~lm}$ & $50.350 \mathrm{l}-\mathrm{q}$ & $44.200 \mathrm{gh}$ & $22.547 \mathrm{~h}$ & $16.083 \mathrm{i}-\mathrm{q}$ & $10.250 \mathrm{~g}$ & $9.000 e-j$ \\
\hline V88 & $55.196 \mathrm{a}-\mathrm{c}$ & $49.5251-q$ & 27.550 no & 30.946 a-h & 9.529 p-r & $11.750 \mathrm{~b}-\mathrm{g}$ & $6.000 \mathrm{n}-\mathrm{q}$ \\
\hline V89 & $56.879 \mathrm{ab}$ & $43.700 \mathrm{p}-\mathrm{t}$ & $36.400 \mathrm{i}-\mathrm{m}$ & 28.998 a-h & $16.375 \mathrm{i}-\mathrm{p}$ & $11.000 \mathrm{~d}-\mathrm{g}$ & $6.5001-q$ \\
\hline V90 & $25.785 \mathrm{k}-\mathrm{m}$ & $71.900 \mathrm{~d}-\mathrm{f}$ & $51.250 \mathrm{f}$ & $31.089 \mathrm{a}-\mathrm{h}$ & $22.052 \mathrm{a}-\mathrm{g}$ & $10.250 \mathrm{~g}$ & $7.250 \mathrm{j}-\mathrm{o}$ \\
\hline V95 & $26.091 \mathrm{j}-\mathrm{m}$ & $65.775 \mathrm{e}-\mathrm{h}$ & $43.625 \mathrm{gh}$ & 28.817 a-h & 18.484 d-m & $13.500 \mathrm{a}-\mathrm{d}$ & 8.000 h-n \\
\hline V99 & $19.417 \mathrm{~m}$ & $65.400 \mathrm{e}-\mathrm{h}$ & 57.150 ef & $33.735 \mathrm{a}-\mathrm{g}$ & $24.560 \mathrm{a}-\mathrm{f}$ & $11.250 \mathrm{c}-\mathrm{g}$ & $7.500 \mathrm{i}-\mathrm{o}$ \\
\hline $\mathrm{T} 7$ & $58.671 \mathrm{a}$ & $63.575 \mathrm{~g}-\mathrm{i}$ & $25.700 \mathrm{n}-\mathrm{p}$ & $34.681 \mathrm{a}-\mathrm{f}$ & 11.346 o-r & $10.250 \mathrm{~g}$ & $4.750 \mathrm{q}$ \\
\hline T32 & $38.175 \mathrm{e}-\mathrm{i}$ & $40.900 \mathrm{r}-\mathrm{v}$ & 27.025 n-p & $24.411 \mathrm{f}-\mathrm{h}$ & $13.020 \mathrm{~m}-\mathrm{r}$ & $11.500 \mathrm{c}-\mathrm{g}$ & $7.250 \mathrm{j}-\mathrm{o}$ \\
\hline $\mathrm{T} 37$ & 29.177 i-m & $53.075 \mathrm{j}-\mathrm{n}$ & 38.575 h-1 & $23.667 \mathrm{gh}$ & $13.048 \mathrm{~m}-\mathrm{r}$ & $11.000 \mathrm{~d}-\mathrm{g}$ & $7.000 \mathrm{j}-\mathrm{o}$ \\
\hline T65 & $19.888 \mathrm{~m}$ & $47.975 \mathrm{~m}-\mathrm{r}$ & $40.775 \mathrm{~g}-\mathrm{j}$ & 29.787 a-h & $18.719 \mathrm{~d}-\mathrm{m}$ & $11.250 \mathrm{c}-\mathrm{g}$ & $9.750 \mathrm{~d}-\mathrm{h}$ \\
\hline $\mathrm{T} 72$ & $56.603 \mathrm{ab}$ & $57.458 \mathrm{~h}-1$ & $23.475 \mathrm{op}$ & $22.651 \mathrm{~h}$ & $8.624 \mathrm{r}$ & $12.500 \mathrm{a}-\mathrm{g}$ & $6.250 \mathrm{~m}-\mathrm{q}$ \\
\hline T121 & $37.056 \mathrm{e}-\mathrm{j}$ & $36.925 \mathrm{u}-\mathrm{v}$ & $24.575 \mathrm{p}-\mathrm{o}$ & $26.275 \mathrm{~d}-\mathrm{h}$ & 13.274 k-r & $11.500 \mathrm{c}-\mathrm{g}$ & 8.000 h-n \\
\hline V-e1 & $46.664 \mathrm{~b}-\mathrm{e}$ & $52.375 \mathrm{j}-\mathrm{o}$ & 26.200 n-p & $25.162 \mathrm{f}-\mathrm{h}$ & 9.722 p-r & $12.250 \mathrm{a}-\mathrm{g}$ & $7.000 \mathrm{j}-\mathrm{o}$ \\
\hline V-a1 & $17.535 \mathrm{~m}$ & $36.350 \mathrm{uv}$ & $31.675 \mathrm{mn}$ & $32.972 \mathrm{a}-\mathrm{g}$ & $24.077 \mathrm{a}-\mathrm{h}$ & $14.250 \mathrm{ab}$ & $13.000 \mathrm{a}$ \\
\hline V-g1 & $37.879 \mathrm{e}-\mathrm{i}$ & $38.175 \mathrm{uv}$ & 25.925 n-p & 29.169 a-h & $14.776 \mathrm{j}-\mathrm{r}$ & $12.000 \mathrm{a}-\mathrm{g}$ & $8.750 \mathrm{f}-\mathrm{k}$ \\
\hline Zulbiye & $59.642 \mathrm{a}$ & $33.950 \mathrm{v}$ & $10.375 \mathrm{q}$ & $23.899 \mathrm{gh}$ & 9.405 p-r & $11.500 \mathrm{c}-\mathrm{g}$ & $5.000 \mathrm{pq}$ \\
\hline Yakutiye & $17.945 \mathrm{~m}$ & $38.650 \mathrm{~s}-\mathrm{V}$ & $34.675 \mathrm{j}-\mathrm{m}$ & $32.006 \mathrm{a}-\mathrm{h}$ & $25.297 \mathrm{a}-\mathrm{d}$ & $13.750 \mathrm{a}-\mathrm{c}$ & $12.000 \mathrm{abc}$ \\
\hline
\end{tabular}

* There is a significant difference $(\mathrm{p}<0.05)$ among the different letters in each column.

in the plant number increased in these genotypes [8]. It was emphasized that high temperatures reduced corn grain yield by $80-90 \%$ compared to normal temperatures [9]. It was reported in a study conducted with two soybean genotypes under high temperature and drought conditions that the decreases in leaf area, seed weight, total biomass, pod, harvest index, seed number per pod and 100 -seed weight were observed [10].

There was a significant difference between the fresh and dry shoot and fresh and dry root weights of tolerant and sensitive common bean plants under high temperature stress. The fresh shoot weight loss was the lowest in the genotype V-al and the highest in the genotype T7. The dry shoot weight was the lowest in the genotype $\mathrm{V}-\mathrm{a} 1$ and the highest in the genotype T7. The fresh root weight lost was the lowest in the genotype $\mathrm{V}$-al and the highest in the genotype T7. The dry root weight was the lowest in the genotype $\mathrm{V}$-al and the highest in the genotype T7 (Table 3). When the fresh and dry shoot/root ratio of bean genotypes under high temperature stress was examined, it was determined that 
Table 3. The fresh and dry shoot and root weights (g) of the bean genotypes in high temperature stress study.

\begin{tabular}{|c|c|c|c|c|c|c|c|c|}
\hline Genotype & $\begin{array}{l}\text { Shoot fresh } \\
\text { weights in } \\
\text { control }(\mathrm{g})\end{array}$ & $\begin{array}{l}\text { Shoot fresh } \\
\text { weights in high } \\
\text { temperature } \\
\text { stress (g) }\end{array}$ & $\begin{array}{l}\text { Shoot dry } \\
\text { weights in } \\
\text { control (g) }\end{array}$ & $\begin{array}{c}\text { Shoot dry } \\
\text { weights in high } \\
\text { temperature } \\
\text { stress }(\mathrm{g})\end{array}$ & $\begin{array}{l}\text { Root fresh } \\
\text { weights in } \\
\text { control (g) }\end{array}$ & $\begin{array}{l}\text { Root fresh } \\
\text { weights in high } \\
\text { temperature } \\
\text { stress }(\mathrm{g})\end{array}$ & $\begin{array}{c}\text { Root dry } \\
\text { weights in } \\
\text { control }(g)\end{array}$ & $\begin{array}{l}\text { Root dry } \\
\text { weights in } \\
\text { drought } \\
\text { stress (g) }\end{array}$ \\
\hline V5 & 26.838 a-g* & 13.187 e-m* & 3.859 p-s* & $2.823 \mathrm{~h}-\mathrm{j} *$ & $6.665 \mathrm{gh}^{*}$ & 3.580 h-k* & $0.901 \mathrm{ab}^{*}$ & $0.571 \mathrm{e}^{*}$ \\
\hline V6 & 24.394 c-I & $16.738 \mathrm{~b}-\mathrm{i}$ & $3.523 \mathrm{suu}$ & $2.844 \mathrm{~g}-\mathrm{j}$ & $5.005 \mathrm{n}$ & $2.250 \mathrm{q}-\mathrm{u}$ & $0.909 \mathrm{ab}$ & $0.462 \mathrm{gh}$ \\
\hline V7 & $25.076 \mathrm{c}-\mathrm{I}$ & $10.2191-p$ & $5.305 \mathrm{efg}$ & 2.743 h-k & $6.040 \mathrm{ijk}$ & $4.881 \mathrm{f}$ & $0.840 \mathrm{~cd}$ & $0.701 \mathrm{~d}$ \\
\hline V10 & $23.808 \mathrm{c}-\mathrm{I}$ & $16.560 \mathrm{~b}-\mathrm{i}$ & $3.636 r-\mathrm{r}$ & $3.131 \mathrm{f}-\mathrm{i}$ & 4.649 nop & $4.088 \mathrm{~h}$ & $0.549 \mathrm{i}-\mathrm{m}$ & $0.393 \mathrm{jk}$ \\
\hline V12 & $33.560 \mathrm{a}$ & $12.073 \mathrm{i}-\mathrm{o}$ & $4.911 \mathrm{hi}$ & $4.246 \mathrm{~b}-\mathrm{d}$ & 3.767 qrs & $3.311 \mathrm{jkl}$ & 0.479 nop & $0.417 \mathrm{ijk}$ \\
\hline V14 & 25.532 b-I & $16.309 \mathrm{~b}-\mathrm{i}$ & $3.194 \mathrm{uv}$ & $2.591 \mathrm{i}-\mathrm{k}$ & 4.702 no & $4.104 \mathrm{~h}$ & $0.523 \mathrm{k}-\mathrm{n}$ & $0.472 \mathrm{fg}$ \\
\hline V15 & 20.443 hi & 13.420 e-m & 2.844 xyz & $2.367 \mathrm{j}-1$ & $3.381 \mathrm{st}$ & $3.111 \mathrm{k}-\mathrm{n}$ & 0.417 qrs & $0.387 \mathrm{k}$ \\
\hline V21 & $25.071 \mathrm{c}-\mathrm{I}$ & $17.024 \mathrm{~b}-\mathrm{i}$ & 4.618 jil & $3.796 \mathrm{c}-\mathrm{e}$ & $8.228 \mathrm{c}$ & $7.306 \mathrm{~d}$ & $0.903 \mathrm{ab}$ & $0.796 \mathrm{~b}$ \\
\hline V28 & $27.298 \mathrm{a}-\mathrm{h}$ & $16.576 \mathrm{~b}-\mathrm{i}$ & 3.996 opq & $2.496 \mathrm{j}-1$ & $5.180 \ln$ & $4.661 \mathrm{~g}$ & 0.599 ghi & $0.496 \mathrm{f}$ \\
\hline V29 & 26.394 b-I & 18.263 a-e & $6.736 \mathrm{bc}$ & $5.247 \mathrm{a}$ & $9.331 \mathrm{~b}$ & $8.607 \mathrm{~b}$ & $0.871 \mathrm{bc}$ & $0.775 \mathrm{bc}$ \\
\hline V30 & $19.491 \mathrm{I}$ & 7.449 op & $2.603 \mathrm{z}$ & $1.626 \mathrm{~m}-\mathrm{o}$ & $4.076 \mathrm{pqr}$ & $1.925 \mathrm{~s}-\mathrm{V}$ & $0.496 \mathrm{~m}-\mathrm{p}$ & $0.191 \mathrm{rst}$ \\
\hline V32 & $32.569 \mathrm{ab}$ & $20.473 \mathrm{ab}$ & $4.3711-\mathrm{o}$ & $3.684 \mathrm{~d}-\mathrm{f}$ & $10.236 \mathrm{a}$ & $9.580 \mathrm{a}$ & $0.940 \mathrm{a}$ & $0.873 \mathrm{a}$ \\
\hline V33 & 24.384 c-I & $11.066 \mathrm{j}-\mathrm{p}$ & $6.100 \mathrm{~d}$ & $4.324 \mathrm{cb}$ & $6.829 \mathrm{fgh}$ & $5.788 \mathrm{e}$ & $0.766 \mathrm{e}$ & $0.703 \mathrm{~d}$ \\
\hline V36 & $27.016 \mathrm{a}-\mathrm{h}$ & $12.282 \mathrm{~h}-\mathrm{o}$ & $4.051 \mathrm{n}-\mathrm{q}$ & $2.646 \mathrm{i}-\mathrm{k}$ & $5.724 \mathrm{jkl}$ & $2.9981-0$ & $0.664 \mathrm{f}$ & $0.422 \mathrm{ij}$ \\
\hline V40 & $25.051 \mathrm{c}-\mathrm{I}$ & $16.821 \mathrm{~b}-\mathrm{i}$ & $5.045 \mathrm{~g}-\mathrm{i}$ & $4.439 \mathrm{~b}$ & $5.020 \mathrm{n}$ & $4.673 \mathrm{~g}$ & $0.5081-0$ & $0.462 \mathrm{gh}$ \\
\hline V41 & 24.579 c-I & $10.0561-\mathrm{p}$ & $3.243 \mathrm{u}-\mathrm{w}$ & $2.335 \mathrm{j}-\mathrm{k}$ & 4.542 nop & $2.316 \mathrm{q}-\mathrm{u}$ & $0.403 \mathrm{rs}$ & $0.205 \mathrm{r}$ \\
\hline V43 & $22.583 \mathrm{f}-\mathrm{I}$ & $9.102 \mathrm{~m}-\mathrm{p}$ & $3.276 \mathrm{t}-\mathrm{v}$ & $1.238 \mathrm{o}$ & $7.221 \mathrm{efg}$ & $2.8901-p$ & $0.821 \mathrm{~d}$ & $0.238 \mathrm{pq}$ \\
\hline V47 & $24.173 c-I$ & $10.2951-p$ & $2.806 \mathrm{y}-\mathrm{z}$ & $1.144 \mathrm{o}$ & $4.252 \mathrm{opq}$ & $2.354 \mathrm{p}-\mathrm{t}$ & $0.302 \mathrm{tu}$ & $0.144 \mathrm{u}-\mathrm{x}$ \\
\hline V49 & $27.466 \mathrm{a}-\mathrm{h}$ & 17.366 a-h & $4.219 \mathrm{~m}-\mathrm{p}$ & $2.277 \mathrm{j}-\mathrm{k}$ & $5.689 \mathrm{klm}$ & $2.8941-p$ & $0.617 \mathrm{~g}$ & 0.277 no \\
\hline V57 & $28.332 \mathrm{a}-\mathrm{g}$ & $15.151 \mathrm{c}-1$ & $3.573 \mathrm{~s}-\mathrm{u}$ & $2.167 \mathrm{k}-\mathrm{m}$ & $3.733 \mathrm{qrs}$ & $1.803 \mathrm{uvw}$ & 0.289 tuv & 0.167 tuv \\
\hline V62 & 25.970 b-I & $13.581 \mathrm{~d}-\mathrm{m}$ & $4.104 n-p$ & $2.433 \mathrm{j}-1$ & 6.304 hij & 3.669 hij & $0.734 \mathrm{e}$ & 0.433 hi \\
\hline V63 & 25.808 b-I & $11.177 \mathrm{j}-\mathrm{p}$ & $2.892 \mathrm{w}-\mathrm{Z}$ & $1.001 \mathrm{o}$ & $5.015 \mathrm{n}$ & $2.614 \mathrm{n}-\mathrm{q}$ & $0.516 \mathrm{lmn}$ & $0.301 \mathrm{mn}$ \\
\hline V64 & $25.321 \mathrm{c}-\mathrm{I}$ & $18.732 \mathrm{a}-\mathrm{d}$ & $4.667 \mathrm{i}-1$ & $3.421 \mathrm{e}-\mathrm{g}$. & $6.547 \mathrm{hi}$ & $5.778 \mathrm{e}$ & 0.596 ghi & $0.421 \mathrm{ij}$ \\
\hline V66 & $28.573 \mathrm{a}-\mathrm{f}$ & 17.425 a-h & $4.247 \mathrm{~m}-\mathrm{o}$ & $3.432 \mathrm{e}-\mathrm{g}$ & 4.799 no & $2.780 \mathrm{~m}-\mathrm{q}$ & $0.571 \mathrm{~g}-\mathrm{k}$ & $0.407 \mathrm{ijk}$ \\
\hline V69 & $30.306 \mathrm{a}-\mathrm{d}$ & $17.240 \mathrm{~b}-\mathrm{i}$ & $5.451 \mathrm{ef}$ & $3.756 \mathrm{de}$ & $8.921 \mathrm{~b}$ & $7.992 \mathrm{c}$ & $0.884 \mathrm{bc}$ & $0.756 \mathrm{c}$ \\
\hline V71 & 26.045 b-I & $13.775 \mathrm{c}-\mathrm{m}$ & 2.989 v-y & $2.259 \mathrm{j}-\mathrm{k}$ & $7.808 \mathrm{~cd}$ & 3.681 hij & $0.681 \mathrm{f}$ & 0.259 op \\
\hline V73 & $28.620 \mathrm{a}-\mathrm{g}$ & $16.445 \mathrm{~b}-\mathrm{i}$ & $4.797 \mathrm{i}-\mathrm{j}$ & $2.259 \mathrm{j}-\mathrm{k}$ & 4.653 nop & $1.936 \mathrm{r}-\mathrm{v}$ & $0.535 \mathrm{j}-\mathrm{m}$ & $0.260 \mathrm{op}$ \\
\hline V77 & $27.936 \mathrm{a}-\mathrm{g}$ & $16.837 \mathrm{~b}-\mathrm{i}$ & $6.805 \mathrm{bc}$ & $4.247 \mathrm{~b}-\mathrm{d}$ & $6.476 \mathrm{hi}$ & $3.530 \mathrm{ijk}$ & $0.582 \mathrm{~g}-\mathrm{j}$ & 0.247 op \\
\hline V79 & $25.572 \mathrm{c}-\mathrm{I}$ & $13.262 \mathrm{e}-\mathrm{m}$ & $3.283 \mathrm{v}-\mathrm{u}$ & $1.9741-n$ & $4.959 \mathrm{n}$ & $2.292 \mathrm{q}-\mathrm{u}$ & $0.255 \mathrm{uv}$ & 0.149 uvw \\
\hline V80 & $27.045 \mathrm{a}-\mathrm{h}$ & 12.444 h-o & $4.223 \mathrm{~m}-\mathrm{p}$ & $2.130 \mathrm{k}-\mathrm{m}$ & 4.871 no & 2.476 o-r & $0.262 \mathrm{uv}$ & $0.130 \mathrm{wx}$ \\
\hline V82 & $22.238 \mathrm{~g}-\mathrm{I}$ & $15.827 \mathrm{~b}-\mathrm{k}$ & $6.927 \mathrm{~b}$ & $5.727 \mathrm{a}$ & $6.463 \mathrm{hi}$ & $6.091 \mathrm{e}$ & $0.742 \mathrm{e}$ & $0.679 \mathrm{~d}$ \\
\hline V86 & $23.826 \mathrm{c}-\mathrm{I}$ & $18.153 \mathrm{a}-\mathrm{f}$ & 4.793 i-k & 3.646 ef & $3.524 \mathrm{rst}$ & $3.263 \mathrm{j}-\mathrm{m}$ & $0.316 \mathrm{t}$ & $0.296 \mathrm{mn}$ \\
\hline V88 & $26.975 \mathrm{a}-\mathrm{h}$ & $9.134 \mathrm{~m}-\mathrm{p}$ & $5.203 \mathrm{f}-\mathrm{h}$ & $1.198 \mathrm{o}$ & 4.783 no & 3.694 hij & 0.509 1-o & $0.198 \mathrm{rs}$ \\
\hline V89 & $25.761 \mathrm{e}-\mathrm{I}$ & $8.012 n-p$ & $5.601 \mathrm{e}$ & $1.9371-n$ & 4.740 no & $1.402 \mathrm{w}$ & 0.556 h-1 & $0.137 \mathrm{vwx}$ \\
\hline V90 & $25.164 \mathrm{c}-\mathrm{I}$ & $17.804 \mathrm{a}-\mathrm{f}$ & $4.912 \mathrm{i}-\mathrm{j}$ & $3.114 \mathrm{f}-\mathrm{i}$ & 3.736 qrs & $2.314 \mathrm{q}-\mathrm{u}$ & $0.237 \mathrm{v}$ & $0.114 \mathrm{x}$ \\
\hline V95 & $28.852 \mathrm{a}-\mathrm{g}$ & $20.513 \mathrm{ab}$ & $7.412 \mathrm{a}$ & $5.438 \mathrm{a}$ & $5.110 \mathrm{mn}$ & $3.583 \mathrm{~h}-\mathrm{k}$ & 0.471 nop & 0.438 hi \\
\hline V99 & 29.847 a-e & $18.806 \mathrm{a}-\mathrm{c}$ & 4.407 1-n & $3.428 \mathrm{e}-\mathrm{g}$ & $3.119 \mathrm{t}$ & $2.774 \mathrm{~m}-\mathrm{q}$ & $0.451 \mathrm{pqr}$ & $0.428 \mathrm{i}$ \\
\hline $\mathrm{T} 7$ & 22.844 e-I & $6.179 \mathrm{p}$ & $4.522 \mathrm{k}-\mathrm{m}$ & $1.139 \mathrm{o}$ & $4.925 \mathrm{n}$ & $1.381 \mathrm{w}$ & $0.382 \mathrm{~s}$ & 0.139 vwx \\
\hline
\end{tabular}


Table 3. Continued.

\begin{tabular}{|c|c|c|c|c|c|c|c|c|}
\hline T32 & $27.580 \mathrm{a}-\mathrm{g}$ & $16.019 \mathrm{~b}-\mathrm{j}$ & $3.682 \mathrm{r}-\mathrm{s}$ & $1.213 \mathrm{o}$ & $5.020 \mathrm{n}$ & $2.436 \mathrm{p}-\mathrm{s}$ & $0.459 \mathrm{opq}$ & $0.213 \mathrm{qr}$ \\
\hline T37 & $27.112 \mathrm{a}-\mathrm{h}$ & $17.411 \mathrm{a}-\mathrm{h}$ & $2.990 \mathrm{v}-\mathrm{y}$ & $1.518 \mathrm{no}$ & $3.815 \mathrm{qrs}$ & $1.848 \mathrm{t}-\mathrm{w}$ & $0.372 \mathrm{~s}$ & $0.174 \mathrm{stu}$ \\
\hline T65 & $23.404 \mathrm{~d}-\mathrm{I}$ & $17.019 \mathrm{~b}-\mathrm{i}$ & $3.531 \mathrm{~s}-\mathrm{u}$ & $2.874 \mathrm{~h}-\mathrm{j}$ & $3.849 \mathrm{qrs}$ & $3.542 \mathrm{ijk}$ & $0.297 \mathrm{tu}$ & $0.264 \mathrm{op}$ \\
\hline T72 & $25.076 \mathrm{c}-\mathrm{I}$ & $7.533 \mathrm{op}$ & $4.923 \mathrm{i}-\mathrm{j}$ & $1.322 \mathrm{o}$ & $3.708 \mathrm{qrs}$ & $1.675 \mathrm{vw}$ & $0.279 \mathrm{tuv}$ & $0.122 \mathrm{wx}$ \\
\hline T121 & $26.857 \mathrm{a}-\mathrm{h}$ & $10.911 \mathrm{k}-\mathrm{p}$ & $5.187 \mathrm{f}-\mathrm{g}$ & $3.318 \mathrm{e}-\mathrm{h}$ & $6.623 \mathrm{hi}$ & $3.358 \mathrm{i}-\mathrm{l}$ & $0.590 \mathrm{ghi}$ & $0.318 \mathrm{~m}$ \\
\hline V-e1 & $30.771 \mathrm{a}-\mathrm{c}$ & $13.025 \mathrm{f}-\mathrm{n}$ & $6.481 \mathrm{c}$ & $2.259 \mathrm{j}-\mathrm{k}$ & $5.796 \mathrm{jk}$ & $2.649 \mathrm{n}-\mathrm{q}$ & $0.457 \mathrm{opq}$ & $0.259 \mathrm{op}$ \\
\hline V-a1 & $29.642 \mathrm{a}-\mathrm{f}$ & $22.312 \mathrm{a}$ & $3.729 \mathrm{r}-\mathrm{s}$ & $3.252 \mathrm{e}-\mathrm{h}$ & $5.634 \mathrm{klm}$ & $5.316 \mathrm{f}$ & $0.5021-\mathrm{p}$ & $0.477 \mathrm{fg}$ \\
\hline V-g1 & $26.636 \mathrm{a}-\mathrm{h}$ & $12.749 \mathrm{~g}-\mathrm{n}$ & $4.689 \mathrm{i}-\mathrm{k}$ & $2.352 \mathrm{j}-\mathrm{k}$ & $7.303 \mathrm{def}$ & $3.860 \mathrm{hi}$ & $0.745 \mathrm{e}$ & 0.3521 \\
\hline Zulbiye & $26.081 \mathrm{~b}-\mathrm{I}$ & $7.409 \mathrm{op}$ & $6.055 \mathrm{~d}$ & $2.327 \mathrm{j}-\mathrm{k}$ & $5.175 \mathrm{lmn}$ & $1.500 \mathrm{vw}$ & $0.5161-\mathrm{n}$ & $0.127 \mathrm{wx}$ \\
\hline Yakutiye & $28.565 \mathrm{a}-\mathrm{g}$ & $20.700 \mathrm{ab}$ & $4.136 \mathrm{n}-\mathrm{p}$ & $3.598 \mathrm{ef}$ & $7.631 \mathrm{de}$ & $7.141 \mathrm{~d}$ & $0.606 \mathrm{gh}$ & $0.573 \mathrm{e}$ \\
\hline
\end{tabular}

* There is a significant difference $(\mathrm{p}<0.05)$ among the different letters in each column.

Table 4. The fresh and dry shoot and root ratio and Stem diameter $(\mathrm{mm})$ of the bean genotypes in high temperature stress study.

\begin{tabular}{|c|c|c|c|c|c|c|}
\hline Genotype & $\begin{array}{l}\text { Fresh shoot/root } \\
\text { ratio in control }\end{array}$ & $\begin{array}{c}\text { Fresh shoot/root ratio } \\
\text { in high temperature } \\
\text { stress }\end{array}$ & $\begin{array}{l}\text { Dry shoot/root } \\
\text { ratio in control }\end{array}$ & $\begin{array}{c}\text { Dry shoot/root ratio } \\
\text { in high temperature } \\
\text { stress }\end{array}$ & $\begin{array}{l}\text { Stem diameter } \\
\text { in control } \\
(\mathrm{mm})\end{array}$ & $\begin{array}{c}\text { Stem diameter in } \\
\text { high temperature } \\
\text { stress }(\mathrm{mm})\end{array}$ \\
\hline V5 & $0.250 \mathrm{e}-\mathrm{i}^{*}$ & $0.277 \mathrm{e}-1^{*}$ & $0.236 b^{*}$ & $0.203 c^{*}$ & $5.738 \mathrm{a}-\mathrm{d}^{*}$ & $4.588 \mathrm{c}-\mathrm{h}^{*}$ \\
\hline V6 & $0.207 \mathrm{i}-\mathrm{m}$ & $0.136 \mathrm{p}-\mathrm{t}$ & $0.259 \mathrm{a}$ & $0.163 \mathrm{~g}-\mathrm{h}$ & $5.508 \mathrm{a}-\mathrm{g}$ & $4.398 \mathrm{c}-1$ \\
\hline V7 & 0.247 e-j & $0.491 \mathrm{a}$ & 0.159 ef & $0.256 \mathrm{a}$ & $5.793 \mathrm{a}-\mathrm{c}$ & $4.023 \mathrm{~g}-\mathrm{o}$ \\
\hline V10 & $0.196 \mathrm{k}-\mathrm{q}$ & $0.249 \mathrm{f}-\mathrm{o}$ & $0.151 \mathrm{fg}$ & $0.126 \mathrm{ij}$ & $4.995 \mathrm{e}-1$ & $4.543 \mathrm{c}-\mathrm{j}$ \\
\hline V12 & $0.121 \mathrm{st}$ & 0.278 e-1 & $0.098 \mathrm{n}-\mathrm{p}$ & $0.099 \mathrm{k}-\mathrm{n}$ & $5.350 \mathrm{~b}-\mathrm{i}$ & $4.513 c-j$ \\
\hline V14 & $0.1861-q$ & $0.254 \mathrm{f}-\mathrm{n}$ & 0.164 ef & $0.182 \mathrm{~d}-\mathrm{f}$ & $5.238 \mathrm{~b}-\mathrm{k}$ & $4.603 \mathrm{c}-\mathrm{g}$ \\
\hline V15 & $0.165 \mathrm{n}-\mathrm{s}$ & $0.241 \mathrm{~g}-\mathrm{o}$ & $0.147 \mathrm{f}-\mathrm{i}$ & $0.162 \mathrm{~g}-\mathrm{h}$ & 4.5401 & $4.160 \mathrm{f}-\mathrm{n}$ \\
\hline V21 & $0.332 \mathrm{ab}$ & $0.433 \mathrm{abc}$ & $0.196 \mathrm{~d}$ & $0.209 \mathrm{c}$ & $5.143 \mathrm{c}-1$ & 4.770 cde \\
\hline V28 & $0.1911-\mathrm{q}$ & $0.282 \mathrm{e}-\mathrm{k}$ & $0.149 \mathrm{f}-\mathrm{h}$ & $0.198 \mathrm{~cd}$ & $4.600 \mathrm{kl}$ & $4.105 \mathrm{f}-\mathrm{n}$ \\
\hline V29 & $0.363 \mathrm{a}$ & $0.481 \mathrm{a}$ & $0.129 \mathrm{i}-1$ & $0.148 \mathrm{~h}$ & $5.335 \mathrm{~b}-\mathrm{i}$ & $4.838 \mathrm{c}$ \\
\hline V30 & $0.210 \mathrm{i}-\mathrm{n}$ & $0.268 \mathrm{e}-\mathrm{m}$ & $0.191 \mathrm{~d}$ & $0.118 \mathrm{i}-\mathrm{k}$ & $4.648 \mathrm{jkl}$ & $3.953 \mathrm{k}-\mathrm{q}$ \\
\hline V32 & $0.330 \mathrm{abc}$ & $0.470 \mathrm{ab}$ & $0.221 \mathrm{c}$ & $0.243 \mathrm{a}$ & 5.598 a-e & $4.883 \mathrm{bc}$ \\
\hline V33 & $0.283 \mathrm{c}-\mathrm{f}$ & 0.224 g-r & $0.126 \mathrm{j}-\mathrm{m}$ & 0.163 g-h & $5.003 \mathrm{e}-1$ & $4.398 \mathrm{c}-1$ \\
\hline V36 & $0.216 \mathrm{~h}-\mathrm{m}$ & $0.244 \mathrm{~g}-\mathrm{o}$ & $0.165 \mathrm{ef}$ & 0.162 g-h & $5.590 \mathrm{a}-\mathrm{e}$ & $4.563 \mathrm{c}-\mathrm{i}$ \\
\hline V40 & $0.202 \mathrm{i}-\mathrm{o}$ & $0.283 \mathrm{e}-\mathrm{j}$ & 0.101 no & $0.104 \mathrm{j}-\mathrm{m}$ & $4.738 \mathrm{~h}-1$ & $4.175 \mathrm{f}-\mathrm{n}$ \\
\hline V41 & $0.1861-q$ & 0.239 g-o & $0.124 \mathrm{k}-\mathrm{m}$ & $0.088 \mathrm{~m}-\mathrm{p}$ & $5.218 \mathrm{~b}-\mathrm{k}$ & $4.413 \mathrm{c}-\mathrm{k}$ \\
\hline V43 & $0.325 \mathrm{abc}$ & $0.356 \mathrm{cde}$ & $0.251 \mathrm{ab}$ & $0.192 \mathrm{c}-\mathrm{e}$ & $4.708 \mathrm{i}-1$ & 3.535 o-r \\
\hline V47 & $0.178 \mathrm{~m}-\mathrm{r}$ & 0.235 g-p & $0.107 \mathrm{mn}$ & $0.126 \mathrm{i}$ & $5.298 \mathrm{~b}-\mathrm{j}$ & $4.430 \mathrm{c}-\mathrm{k}$ \\
\hline V49 & $0.208 \mathrm{i}-\mathrm{n}$ & $0.167 \mathrm{~m}-\mathrm{t}$ & $0.146 \mathrm{f}-\mathrm{j}$ & $0.121 \mathrm{ij}$ & $5.083 \mathrm{~d}-1$ & 3.998 j-p \\
\hline V57 & 0.133 rst & $0.124 \mathrm{st}$ & 0.081 o-r & 0.077 o-r & $5.020 \mathrm{e}-1$ & 4.060 g-o \\
\hline V62 & $0.245 \mathrm{f}-\mathrm{k}$ & $0.306 \mathrm{~d}-\mathrm{i}$ & $0.179 \mathrm{de}$ & $0.178 \mathrm{~d}-\mathrm{g}$ & $5.390 \mathrm{~b}-\mathrm{h}$ & $4.610 \mathrm{c}-\mathrm{g}$ \\
\hline V63 & $0.1951-q$ & $0.254 \mathrm{f}-\mathrm{n}$ & $0.178 \mathrm{de}$ & $0.231 \mathrm{~b}$ & $5.320 \mathrm{~b}-\mathrm{i}$ & $4.405 \mathrm{c}-\mathrm{k}$ \\
\hline V64 & $0.259 \mathrm{~d}-\mathrm{h}$ & $0.312 \mathrm{~d}-\mathrm{g}$ & $0.128 \mathrm{i}-\mathrm{m}$ & $0.119 \mathrm{i}-\mathrm{k}$ & $4.805 \mathrm{~h}-1$ & $4.160 \mathrm{f}-\mathrm{n}$ \\
\hline V66 & $0.170 \mathrm{~m}-\mathrm{r}$ & $0.160 \mathrm{n}-\mathrm{t}$ & $0.135 \mathrm{f}-\mathrm{j}$ & $0.119 \mathrm{i}-\mathrm{k}$ & $5.480 \mathrm{a}-\mathrm{g}$ & $4.623 \mathrm{c}-\mathrm{f}$ \\
\hline V69 & $0.298 \mathrm{bcd}$ & $0.505 \mathrm{a}$ & $0.162 \mathrm{ef}$ & $0.159 \mathrm{gh}$ & $5.763 \mathrm{abc}$ & $4.888 \mathrm{bc}$ \\
\hline
\end{tabular}


Table 4. Continued.

\begin{tabular}{|c|c|c|c|c|c|c|}
\hline V71 & $0.300 \mathrm{bcd}$ & $0.271 \mathrm{e}-1$ & $0.229 \mathrm{c}$ & $0.115 \mathrm{i}-1$ & $5.578 \mathrm{a}-\mathrm{f}$ & $4.368 \mathrm{c}-1$ \\
\hline V73 & $0.163 \mathrm{n}-\mathrm{s}$ & $0.120 \mathrm{st}$ & $0.1121-n$ & 0.115 i-1 & $5.038 \mathrm{e}-1$ & $4.145 \mathrm{f}-\mathrm{n}$ \\
\hline V77 & $0.233 \mathrm{~g}-1$ & $0.203 \mathrm{j}-\mathrm{t}$ & $0.085 \mathrm{o}-\mathrm{q}$ & $0.058 \mathrm{r}$ & $5.323 \mathrm{~b}-\mathrm{i}$ & $4.273 \mathrm{~d}-\mathrm{m}$ \\
\hline V79 & 0.198 k-p & $0.1801-\mathrm{t}$ & 0.077 p-r & 0.076 o-r & 4.790 h-1 & $3.728 \mathrm{n}-\mathrm{r}$ \\
\hline V80 & $0.1851-q$ & $0.208 \mathrm{~h}-\mathrm{t}$ & $0.063 \mathrm{t}-\mathrm{r}$ & $0.062 \mathrm{qr}$ & $5.088 \mathrm{~d}-1$ & 4.220 e-n \\
\hline V82 & $0.295 \mathrm{~b}-\mathrm{e}$ & $0.387 \mathrm{bcd}$ & $0.107 \mathrm{mn}$ & $0.119 \mathrm{i}-\mathrm{k}$ & $5.020 \mathrm{e}-1$ & $4.543 \mathrm{c}-\mathrm{j}$ \\
\hline V86 & $0.151 \mathrm{o}-\mathrm{t}$ & $0.180 \mathrm{k}-\mathrm{t}$ & $0.066 \mathrm{r}-\mathrm{t}$ & $0.081 \mathrm{n}-\mathrm{q}$ & 4.968 e-1 & $4.475 \mathrm{c}-\mathrm{k}$ \\
\hline V88 & $0.181 \mathrm{~m}-\mathrm{r}$ & $0.414 \mathrm{abc}$ & 0.097 n-p & $0.169 \mathrm{f}-\mathrm{h}$ & $5.345 \mathrm{~b}-\mathrm{i}$ & $3.493 \mathrm{pqr}$ \\
\hline V89 & $0.1901-q$ & $0.193 \mathrm{j}-\mathrm{t}$ & 0.099 no & 0.071 o-r & $4.908 \mathrm{~g}-1$ & $3.353 \mathrm{r}$ \\
\hline V90 & $0.149 \mathrm{p}-\mathrm{t}$ & $0.133 \mathrm{q}-\mathrm{t}$ & $0.048 \mathrm{t}$ & $0.037 \mathrm{~s}$ & $5.268 \mathrm{~b}-\mathrm{k}$ & $4.350 \mathrm{c}-1$ \\
\hline V95 & $0.177 \mathrm{~m}-\mathrm{r}$ & $0.1741-\mathrm{t}$ & $0.057 \mathrm{st}$ & $0.081 \mathrm{n}-\mathrm{q}$ & $5.385 \mathrm{~b}-\mathrm{h}$ & $4.620 \mathrm{c}-\mathrm{f}$ \\
\hline V99 & $0.104 \mathrm{t}$ & $0.148 \mathrm{o}-\mathrm{t}$ & 0.102 no & $0.125 \mathrm{ij}$ & $5.410 \mathrm{~b}-\mathrm{h}$ & $4.780 \mathrm{~cd}$ \\
\hline $\mathrm{T} 7$ & $0.217 \mathrm{~h}-\mathrm{m}$ & $0.227 \mathrm{~g}-\mathrm{q}$ & $0.085 \mathrm{o}-\mathrm{q}$ & $0.122 \mathrm{ij}$ & $5.180 \mathrm{c}-1$ & $3.398 \mathrm{r}$ \\
\hline T32 & $0.183 \mathrm{l}-\mathrm{q}$ & $0.168 \mathrm{~m}-\mathrm{t}$ & $0.1251-\mathrm{m}$ & $0.176 \mathrm{e}-\mathrm{g}$ & $4.910 \mathrm{~g}-1$ & $4.203 \mathrm{f}-\mathrm{n}$ \\
\hline $\mathrm{T} 37$ & $0.146 \mathrm{rst}$ & $0.111 \mathrm{t}$ & $0.1251-\mathrm{m}$ & 0.115 i-1 & $5.193 \mathrm{~b}-1$ & $4.135 \mathrm{f}-\mathrm{n}$ \\
\hline T65 & $0.168 \mathrm{~m}-\mathrm{s}$ & 0.220 g-r & $0.084 \mathrm{o}-\mathrm{q}$ & $0.092 \mathrm{~m}-\mathrm{o}$ & $4.843 \mathrm{~g}-1$ & $4.380 \mathrm{c}-1$ \\
\hline $\mathrm{T} 72$ & $0.149 \mathrm{p}-\mathrm{t}$ & $0.247 \mathrm{f}-\mathrm{o}$ & $0.057 \mathrm{st}$ & 0.092 1-o & $5.225 \mathrm{~b}-\mathrm{k}$ & 3.543 o-r \\
\hline T121 & $0.249 \mathrm{e}-\mathrm{i}$ & $0.309 \mathrm{~d}-\mathrm{h}$ & $0.114 \mathrm{k}-\mathrm{n}$ & 0.096 1-o & $5.025 \mathrm{e}-1$ & $4.043 \mathrm{~g}-\mathrm{o}$ \\
\hline V-e1 & $0.1911-q$ & $0.212 \mathrm{~g}-\mathrm{s}$ & $0.071 \mathrm{q}-\mathrm{s}$ & $0.115 \mathrm{i}-1$ & $5.250 \mathrm{~b}-\mathrm{k}$ & $3.8531-\mathrm{r}$ \\
\hline V-a1 & $0.1901-q$ & $0.239 \mathrm{~g}-\mathrm{o}$ & $0.135 \mathrm{~g}-\mathrm{k}$ & $0.147 \mathrm{~h}$ & $5.853 \mathrm{ab}$ & $5.333 \mathrm{ab}$ \\
\hline V-g1 & $0.288 \mathrm{~b}-\mathrm{f}$ & $0.307 \mathrm{~d}-\mathrm{i}$ & 0.159 ef & $0.149 \mathrm{~h}$ & $5.140 \mathrm{c}-1$ & $3.743 \mathrm{~m}-\mathrm{r}$ \\
\hline Zulbiye & $0.2001-p$ & $0.207 \mathrm{i}-\mathrm{t}$ & $0.085 \mathrm{o}-\mathrm{q}$ & $0.067 \mathrm{qr}$ & $5.283 \mathrm{~b}-\mathrm{j}$ & $3.448 \mathrm{qr}$ \\
\hline Yakutiye & $0.267 \mathrm{~d}-\mathrm{g}$ & $0.346 \mathrm{c}-\mathrm{f}$ & $0.147 \mathrm{~g}-\mathrm{i}$ & $0.160 \mathrm{gh}$ & $6.078 \mathrm{a}$ & $5.625 \mathrm{a}$ \\
\hline
\end{tabular}

* There is a significant difference $(\mathrm{p}<0.05)$ among the different letters in each column.

the fresh shoot/root ratio varied between 0.111 and 0.505 , while the dry shoot/root ratio varied between 0.058 and 0.243 (Table 4). The stem diameter of the genotype $\mathrm{V}$-al was the least affected by high temperature stress, while the most affected genotype was T7 (Table 4). It was reported that the direct exposure of the mung bean genotypes to high temperatures $\left(45^{\circ} \mathrm{C} / 30^{\circ} \mathrm{C}\right)$ led to about $9 \%$ to $41 \%$ decreases in the mean shoot biomass (including root), and there were significant differences between the genotypes based on root weight; however, there was also a positive correlation between root and shoot weight [6]. In a study where peanut genotypes were analyzed based on pod yield and physiological parameters under heat stress and in environments without stress, it was reported that pod yield, 100-seed weight and pod growth rate could be used as the criteria in selection of heat tolerant genotypes and based on stress tolerance index and pod yield performance, ICGVs 07246, 07012, 06039, 06040, 03042, 07038 and 06424 were described as heat-tolerant genotypes [11]. In a study investigating the effects of high temperature stress on yield in pepper genotypes, it was reported that the optimum temperature for flowering and fruiting of pepper was between $20.70^{\circ} \mathrm{C}$ and $30,74^{\circ} \mathrm{C}$, and temperatures between $24.22^{\circ} \mathrm{C}$ and $32.17^{\circ} \mathrm{C}$ would significantly affect pepper growth and yield [12].

Analysis of the variations in shoot potassium content in bean genotypes exposed to high temperature stress demonstrated that the lowest changes were observed in the genotype V99 and the changes were also significant in the genotypes V29 and V-a1. The least affected one was cv. Yakutiye (Table 5). Among the sensitive cultivars, high $\mathrm{K}$ losses were observed in the genotypes V80, V88, and T7 and cv. Zulbiye. Analysis of the variations in root $\mathrm{K}$ content in bean genotypes exposed to high-temperature stress demonstrated that the lowest changes were observed in the genotypes V82, V95, $\mathrm{V}$-al and V79. The maximum reduction in $\mathrm{K}$ content was determined in the genotypes V89 and T7 (Table 5).

The genotypes with the lowest loss in shoot $\mathrm{Ca}$ content were V14, V-a1, V82, and V15 when exposed to high temperature stress. It was observed that $\mathrm{Ca}$ content in cv. Yakutiye's shoots was $2.938 \%$ in the control group and $2.572 \%$ in the high temperature stress 
Table 5. The fresh and dry shoot and root $\mathrm{K}$ and $\mathrm{Ca}(\%)$ of the bean genotypes in the high temperature stress study.

\begin{tabular}{|c|c|c|c|c|c|c|c|c|}
\hline Genotype & $\begin{array}{c}\text { Shoot K } \\
\text { content in } \\
\text { control (\%) }\end{array}$ & $\begin{array}{c}\text { Shoot K } \\
\text { content in high } \\
\text { temperature } \\
\text { stress }(\%)\end{array}$ & $\begin{array}{c}\text { Root K } \\
\text { content } \\
\text { in control } \\
(\%)\end{array}$ & \begin{tabular}{|c|} 
Root K \\
content in high \\
temperature \\
stress $(\%)$
\end{tabular} & $\begin{array}{l}\text { Shoot Ca } \\
\text { content in } \\
\text { control (\%) }\end{array}$ & \begin{tabular}{|c} 
Shoot $\mathrm{Ca}$ \\
content in high \\
temperature \\
stress $(\%)$
\end{tabular} & $\begin{array}{c}\text { Root } \mathrm{Ca} \\
\text { content } \\
\text { in control } \\
(\%)\end{array}$ & \begin{tabular}{|c} 
Root $\mathrm{Ca}$ \\
content in high \\
temperature \\
stress (\%)
\end{tabular} \\
\hline V5 & $2.399 \mathrm{de}^{*}$ & $1.942 \mathrm{ef}^{*}$ & $1.805 \mathrm{e}^{*}$ & $1.449 \mathrm{~g}^{*}$ & $3.299 \mathrm{c}^{*}$ & $2.325 \mathrm{ij} *$ & $2.163 \mathrm{~g}^{*}$ & $1.621 \mathrm{i}^{*}$ \\
\hline V6 & $1.787 \mathrm{z}$ & $1.510 \mathrm{op}$ & $1.233 \mathrm{v}$ & $1.028 \mathrm{v}$ & $3.042 \mathrm{j}-\mathrm{k}$ & $2.291 \mathrm{j}$ & $1.467 \mathrm{u}$ & $1.195 \mathrm{u}$ \\
\hline V7 & $2.018 \mathrm{wx}$ & $1.197 \mathrm{t}$ & $1.657 \mathrm{kl}$ & $1.443 \mathrm{~g}$ & $2.682 \mathrm{r}$ & $2.029 \mathrm{q}$ & $1.819 \mathrm{n}$ & $1.138 \mathrm{w}$ \\
\hline V10 & $2.375 \mathrm{ef}$ & $2.059 \mathrm{ab}$ & $1.755 \mathrm{f}-\mathrm{h}$ & $1.122 \mathrm{q}$ & $2.714 \mathrm{qr}$ & $2.235 \mathrm{k}$ & $2.031 \mathrm{i}$ & $1.780 \mathrm{~g}$ \\
\hline V12 & $2.386 \mathrm{e}$ & $1.865 \mathrm{gh}$ & $1.591 \mathrm{n}$ & $1.248 \mathrm{~m}$ & $3.229 \mathrm{de}$ & $2.408 \mathrm{~h}$ & $1.218 \mathrm{w}$ & $0.936 \mathrm{yz}$ \\
\hline V14 & $2.208 \mathrm{o}-\mathrm{t}$ & $1.765 \mathrm{ji}$ & $1.656 \mathrm{kl}$ & $1.075 \mathrm{t}$ & $3.384 \mathrm{ab}$ & $3.034 \mathrm{a}$ & $1.693 \mathrm{r}$ & $1.465 \mathrm{~m}$ \\
\hline V15 & $1.758 \mathrm{z}$ & $1.498 \mathrm{op}$ & $1.382 \mathrm{r}$ & $1.085 \mathrm{st}$ & $2.683 \mathrm{r}$ & $2.362 \mathrm{i}$ & $2.388 \mathrm{c}$ & $2.112 \mathrm{~b}$ \\
\hline V21 & $2.447 \mathrm{bc}$ & $1.992 \mathrm{c}-\mathrm{e}$ & $1.758 \mathrm{fg}$ & $1.395 \mathrm{~h}$ & 2.727 p-r & $2.298 \mathrm{j}$ & 1.9131 & $1.597 \mathrm{j}$ \\
\hline V28 & $2.264 \mathrm{j}-1$ & $1.957 \mathrm{ef}$ & $1.848 \mathrm{~d}$ & $1.369 \mathrm{ij}$ & $3.157 \mathrm{f}-\mathrm{g}$ & $2.545 \mathrm{fg}$ & $1.343 \mathrm{v}$ & $0.907 \mathrm{yz}$ \\
\hline V29 & $2.196 \mathrm{q}-\mathrm{t}$ & $1.939 \mathrm{ef}$ & $1.733 \mathrm{ij}$ & 1.2681 & 3.148 g-i & $2.755 \mathrm{~b}$ & $1.688 \mathrm{r}$ & $1.358 \mathrm{n}$ \\
\hline $\mathrm{V} 30$ & $1.798 \mathrm{z}$ & $1.373 \mathrm{rs}$ & $1.659 \mathrm{k}$ & $0.880 \mathrm{z}$ & 3.190 e-g & $1.968 \mathrm{r}$ & $1.474 \mathrm{u}$ & $0.831 \mathrm{z}$ \\
\hline V32 & $2.392 \mathrm{de}$ & $2.114 \mathrm{a}$ & $1.840 \mathrm{~d}$ & $1.573 \mathrm{c}$ & 3.217 ef & $2.708 \mathrm{c}$ & $2.164 \mathrm{~g}$ & $1.919 \mathrm{~d}$ \\
\hline V33 & $2.203 \mathrm{p}-\mathrm{t}$ & $1.866 \mathrm{gh}$ & $1.543 \mathrm{o}$ & $1.173 \mathrm{o}$ & $2.785 \mathrm{p}$ & $2.346 \mathrm{i}$ & $2.389 \mathrm{c}$ & $1.931 \mathrm{~d}$ \\
\hline V36 & $2.111 \mathrm{v}$ & $1.367 \mathrm{rs}$ & $1.735 \mathrm{~h}-\mathrm{j}$ & $1.108 \mathrm{qr}$ & $3.332 \mathrm{bc}$ & $2.538 \mathrm{fg}$ & $2.557 \mathrm{~b}$ & $1.847 \mathrm{f}$ \\
\hline V40 & $2.478 \mathrm{ab}$ & $2.068 \mathrm{ab}$ & $1.641 \mathrm{kl}$ & $1.456 \mathrm{fg}$ & $3.083 \mathrm{i}-\mathrm{k}$ & $2.752 b$ & $2.339 \mathrm{e}$ & $2.114 \mathrm{~b}$ \\
\hline V41 & $2.489 \mathrm{a}$ & $1.647 \mathrm{~lm}$ & $1.798 \mathrm{e}$ & $1.093 \mathrm{rs}$ & $3.172 \mathrm{e}-\mathrm{g}$ & $2.093 \mathrm{o}$ & $1.489 \mathrm{u}$ & $1.234 \mathrm{~s}$ \\
\hline V43 & $2.283 \mathrm{ij}$ & $1.689 \mathrm{kl}$ & $1.205 \mathrm{w}$ & $0.975 \mathrm{w}$ & $2.718 \mathrm{qr}$ & $1.245 \mathrm{y}$ & $1.601 \mathrm{~s}$ & $0.842 \mathrm{z}$ \\
\hline V47 & $2.244 \mathrm{k}-\mathrm{o}$ & $1.679 \mathrm{kl}$ & $1.408 \mathrm{q}$ & $1.118 \mathrm{q}$ & $2.697 \mathrm{qr}$ & $2.053 \mathrm{pq}$ & $1.995 \mathrm{j}$ & $1.577 \mathrm{k}$ \\
\hline V49 & $2.185 \mathrm{r}-\mathrm{u}$ & 1.528 no & 1.739 g-i & $1.248 \mathrm{~m}$ & $2.763 \mathrm{pq}$ & $2.207 \mathrm{kl}$ & $1.999 \mathrm{j}$ & 1.5121 \\
\hline V57 & 2.222 n-r & 1.939 ef & $1.358 \mathrm{~s}$ & $1.089 \mathrm{st}$ & $2.724 \mathrm{p}-\mathrm{r}$ & $2.164 \mathrm{mn}$ & $2.007 \mathrm{j}$ & $1.339 \mathrm{o}$ \\
\hline V62 & $2.289 \mathrm{ij}$ & 1.947 ef & $1.842 \mathrm{~d}$ & $1.327 \mathrm{k}$ & $3.136 \mathrm{~g}-\mathrm{i}$ & $1.606 \mathrm{u}$ & $2.093 \mathrm{~h}$ & $1.173 \mathrm{v}$ \\
\hline V63 & $1.807 \mathrm{z}$ & $1.408 \mathrm{qr}$ & $1.324 \mathrm{t}$ & $1.059 \mathrm{u}$ & $2.713 \mathrm{qr}$ & $1.730 \mathrm{t}$ & $1.595 \mathrm{~s}$ & $1.197 \mathrm{u}$ \\
\hline V64 & $2.035 \mathrm{w}$ & 1.516 op & $1.235 \mathrm{v}$ & $1.019 \mathrm{v}$ & 2.747 p-r & $2.359 \mathrm{i}$ & $1.718 \mathrm{q}$ & $1.265 \mathrm{r}$ \\
\hline V66 & $2.259 \mathrm{j}-\mathrm{m}$ & $1.941 \mathrm{ef}$ & $1.801 \mathrm{e}$ & $1.505 \mathrm{e}$ & $3.285 \mathrm{~cd}$ & $2.678 \mathrm{c}$ & $1.699 \mathrm{qr}$ & $1.309 \mathrm{pq}$ \\
\hline V69 & $2.443 b c$ & $1.890 \mathrm{fg}$ & $1.601 \mathrm{mn}$ & $1.355 \mathrm{j}$ & $3.227 \mathrm{de}$ & $2.560 \mathrm{e}-\mathrm{g}$ & $1.467 \mathrm{u}$ & $1.149 \mathrm{w}$ \\
\hline V71 & $2.346 \mathrm{fg}$ & 1.954 ef & $1.859 \mathrm{~d}$ & 1.2671 & $2.686 \mathrm{r}$ & $2.028 \mathrm{q}$ & $1.334 \mathrm{v}$ & 1.0561 \\
\hline V73 & $2.093 \mathrm{v}$ & $1.723 \mathrm{jk}$ & $1.859 \mathrm{~d}$ & $1.373 \mathrm{i}$ & $2 . .849 \mathrm{o}$ & $2.185 \mathrm{~lm}$ & $1.474 \mathrm{u}$ & $1.217 \mathrm{t}$ \\
\hline V77 & $2.2341-\mathrm{p}$ & $1.666 \mathrm{kl}$ & 1.741 g-i & $1.225 \mathrm{n}$ & $2.9781-n$ & $1.979 \mathrm{r}$ & $2.260 \mathrm{f}$ & $1.712 \mathrm{~h}$ \\
\hline V79 & $2.171 \mathrm{tu}$ & $1.588 \mathrm{mn}$ & $1.473 \mathrm{p}$ & $1.326 \mathrm{k}$ & $2.858 \mathrm{o}$ & $2.222 \mathrm{kl}$ & $1.521 \mathrm{t}$ & $1.294 \mathrm{q}$ \\
\hline V80 & $2.422 \mathrm{~cd}$ & $1.501 \mathrm{op}$ & $1.949 \mathrm{a}$ & $1.338 \mathrm{k}$ & $2.861 \mathrm{o}$ & $2.101 \mathrm{o}$ & $1.759 p$ & $1.471 \mathrm{~m}$ \\
\hline V82 & $2.279 \mathrm{i}-\mathrm{k}$ & 1.934 ef & $1.812 \mathrm{e}$ & $1.698 \mathrm{~b}$ & $2.531 \mathrm{~s}$ & $2.235 \mathrm{k}$ & $1.696 \mathrm{qr}$ & 1.5091 \\
\hline V86 & $2.366 \mathrm{ef}$ & $1.837 \mathrm{gh}$ & $1.773 \mathrm{f}$ & $1.243 \mathrm{~m}$ & $3.038 \mathrm{j}-1$ & $2.597 \mathrm{e}$ & $1.969 \mathrm{k}$ & 1.4991 \\
\hline V88 & $2.211 \mathrm{n}-\mathrm{s}$ & $1.385 \mathrm{r}$ & $1.912 \mathrm{~b}$ & $0.949 \mathrm{x}$ & 3.144 g-i & $1.514 \mathrm{v}$ & $1.686 \mathrm{r}$ & $0.840 \mathrm{z}$ \\
\hline V89 & $1.985 \mathrm{y}$ & $1.315 \mathrm{~s}$ & 1.6381 & $0.719 \mathrm{z}$ & $3.028 \mathrm{kl}$ & $2.673 \mathrm{~cd}$ & $1.139 \mathrm{y}$ & $0.893 \mathrm{yz}$ \\
\hline V90 & $1.985 \mathrm{xy}$ & 1.6511 & $1.360 \mathrm{~s}$ & $0.925 \mathrm{y}$ & $2.942 n$ & $2.142 n$ & $1.171 \mathrm{x}$ & $0.838 \mathrm{z}$ \\
\hline V95 & $2.449 \mathrm{bc}$ & $1.970 \mathrm{de}$ & $1.949 \mathrm{a}$ & $1.803 \mathrm{a}$ & $3.008 \mathrm{~lm}$ & $2.343 \mathrm{i}$ & $1.595 \mathrm{~s}$ & $1.308 \mathrm{pq}$ \\
\hline V99 & $2.308 \mathrm{hi}$ & $2.048 \mathrm{bc}$ & $1.891 \mathrm{c}$ & $1.698 \mathrm{~b}$ & $2.9831-n$ & $2.443 \mathrm{~h}$ & $2.262 \mathrm{f}$ & $2.122 \mathrm{~b}$ \\
\hline $\mathrm{T} 7$ & $2.177 \mathrm{~s}-\mathrm{u}$ & $1.406 \mathrm{qr}$ & $1.856 \mathrm{~d}$ & $0.885 \mathrm{z}$ & $2.959 \mathrm{mn}$ & $1.474 \mathrm{w}$ & $1.529 \mathrm{t}$ & $0.809 \mathrm{z}$ \\
\hline
\end{tabular}


Table 5. Continued.

\begin{tabular}{|c|c|c|c|c|c|c|c|c|}
\hline T32 & $2.246 \mathrm{k}-\mathrm{n}$ & $1.819 \mathrm{hi}$ & $1.792 \mathrm{e}$ & $1.470 \mathrm{f}$ & $3.395 \mathrm{a}$ & $2.525 \mathrm{~g}$ & $1.469 \mathrm{u}$ & $1.094 \mathrm{x}$ \\
\hline T37 & $2.282 \mathrm{ij}$ & $1.809 \mathrm{hi}$ & $1.224 \mathrm{v}$ & $0.922 \mathrm{y}$ & $3.334 \mathrm{bc}$ & $2.644 \mathrm{~d}$ & $2.718 \mathrm{a}$ & $1.989 \mathrm{c}$ \\
\hline T65 & $2.223 \mathrm{~m}-\mathrm{q}$ & $1.826 \mathrm{~g}-\mathrm{i}$ & $1.255 \mathrm{u}$ & $1.108 \mathrm{qr}$ & $3.318 \mathrm{c}$ & $2.527 \mathrm{~g}$ & $2.362 \mathrm{~d}$ & $2.217 \mathrm{a}$ \\
\hline T72 & $2.156 \mathrm{u}$ & $1.663 \mathrm{kl}$ & $1.455 \mathrm{p}$ & $0.694 \mathrm{~b}$ & $3.126 \mathrm{~g}-\mathrm{i}$ & $1.337 \mathrm{x}$ & $2.282 \mathrm{f}$ & $1.091 \mathrm{y}$ \\
\hline T121 & $2.005 \mathrm{x}-\mathrm{y}$ & $1.450 \mathrm{pq}$ & $1.546 \mathrm{o}$ & $1.138 \mathrm{p}$ & $3.008 \mathrm{~lm}$ & $1.852 \mathrm{~s}$ & $1.864 \mathrm{~m}$ & $1.306 \mathrm{pq}$ \\
\hline V-e1 & $2.195 \mathrm{q}-\mathrm{t}$ & $1.548 \mathrm{no}$ & $1.618 \mathrm{~m}$ & 1.2781 & $3.144 \mathrm{~g}-\mathrm{i}$ & $2.442 \mathrm{x}$ & 1.9231 & $1.321 \mathrm{p}$ \\
\hline V-a1 & $2.311 \mathrm{hi}$ & $2.030 \mathrm{~b}-\mathrm{d}$ & $1.744 \mathrm{~g}-\mathrm{i}$ & $1.578 \mathrm{c}$ & $2.9931-\mathrm{n}$ & $2.646 \mathrm{~d}$ & $2.044 \mathrm{i}$ & $1.859 \mathrm{f}$ \\
\hline V-g1 & $2.328 \mathrm{gh}$ & $1.847 \mathrm{gh}$ & $1.891 \mathrm{c}$ & $1.536 \mathrm{~d}$ & $3.098 \mathrm{~h}-\mathrm{j}$ & $2.083 \mathrm{op}$ & $1.341 \mathrm{v}$ & $1.018 \mathrm{y}$ \\
\hline Zulbiye & $2.290 \mathrm{ij}$ & $1.366 \mathrm{rs}$ & $1.719 \mathrm{j}$ & $0.948 \mathrm{x}$ & $3.139 \mathrm{~g}-\mathrm{i}$ & $1.495 \mathrm{vw}$ & $1.783 \mathrm{o}$ & $0.921 \mathrm{z}$ \\
\hline Yakutiye & $2.173 \mathrm{~s}-\mathrm{u}$ & $1.942 \mathrm{ef}$ & $1.849 \mathrm{~d}$ & $1.701 \mathrm{~b}$ & $2.938 \mathrm{n}$ & $2.572 \mathrm{ef}$ & $2.043 \mathrm{i}$ & $1.904 \mathrm{e}$ \\
\hline
\end{tabular}

* There is a significant difference $(\mathrm{p}<0.05)$ among the different letters in each column.

exposure group (Table 5). The genotypes V41, T7 and T72 genotypes and cv. Zulbiye variety demonstrated the highest $\mathrm{Ca}$ content.

The lowest $\mathrm{Ca}$ losses in plant roots were determined in the genotypes T65, V99, V-a1 and cv. Yakutiye. The highest changes in $\mathrm{Ca}$ content were observed in the genotypes V88, V43 and T7. Increase in temperatures during bean cultivation leads to necrotic injury and alterations in cellular functions, especially in the plant stem and leaves [1-3]. Nutrient intake of the plants depends on root morphology, soil properties, drought and plant species. Drought, extreme temperatures and mineral nutrition are closely related. Higher temperatures affect nutrient intake negatively and reduce mineral intake [4]. If the plant $\mathrm{K}$ and $\mathrm{Ca}$ content is sufficient, transpiration would be under control and the opening and closure of stomata would exhibit a linear correlation with the $\mathrm{K}$ content in specialized stoma cells.

High temperature stress also leads to drought damages since the water intake of the plants is adversely affected. In a study aiming to determine the tolerance and sensitivity of 46 bean genotypes and 2 bean cultivars to drought stress, it was determined that the genotypes V7, V15, V33, V82, V89, and V-A1 and cv. Yakutiye were tolerant and the genotypes V21, V62, V69, V71, V86, V95, and T7 and cv. Zulbiye were sensitive to drought [13]. It was reported that leaf area and number, plant height and weight, stem diameter and relative leaf water content, and $\mathrm{K}$ and $\mathrm{Ca}$ content decreased in the bean genotypes under drought stress, while the membrane damage index increased $[13,15]$. It was indicated that the plant weight, leaf number, stem diameter and relative leaf water content decreased and membrane damage index increased in tomato plants exposed to drought stress [17, 18]. In tomato cultivars exposed to drought stress, the fresh and dry weight, leaf area and relative leaf water content significantly decreased in all cultivars when compared to the control group, while in bean plants, water and high temperature stress adversely affected the growth of sensitive bean cultivars [19]. It has been reported in another study that organic and chemical fertilizers increase the growth and development and nutrient content of vegetable plants under abiotic stress [29-32].

\section{Conclusions}

In the present study conducted to determine high temperature sensitive and tolerant bean genotypes, the changes in $0-5$ visual scale scores, fresh and dry weight, growth rate, fresh and dry shoot/root weight ratios, stem diameter, plant height, leaf number and area, relative leaf water content, membrane damage index, shoot and root ion ( $\mathrm{K}$ and $\mathrm{Ca}$ ) content in 46 genotypes and 2 cultivars were examined. It was determined that the genotypes V21, V62, V69, V71, V86, V95, and T7 and $\mathrm{cv}$. Zulbiye were sensitive to high temperature stress, while the genotypes V7, V15, V33, V82, and V89 and $\mathrm{V}-\mathrm{A} 1$ and $\mathrm{cv}$. Yakutiye were tolerant ones.

In conclusion, the present study once more evidenced that the parameters applied to determine the effects of high temperature stress on bean genotypes were suitable methods in selecting genotypes tolerant to high temperature stress.

\section{Acknowledgments}

This research is a part of $\mathrm{PhD}$ thesis of the first author, and was partially supported by the Yuzuncu Yil University Scientific Research Projects (BAP) Coordination Unit (Project No. 2013-FBD011). We also thank some seed suppliers: Ceknas Erdinc, Huseyin Ozcelik, and Tulay Dizikisa.

\section{Conflict of Interest}

The authors declare no conflict of interest. 


\section{References}

1. KACAR B., KATKAT B., ÖZTÜRK, Ş. Plant Physiology. Nobel Press, 2, 533, 2006.

2. OZEN H.C., ONAY A. Plant Physiology. ISBN 978-605395-017-2, Nobel, 2007.

3. WAHID A., GELAN, S., ASHRAF M., FOOLAD M.R. Heat tolerance in plants: an overview. Environmental and Experimental Botany, 61 (3), 199, 2007.

4. NAM M. Patates Çeşitlerinin Yüksek Sıcaklık Stresine Toleranslarının Büyüme ve Verim Parametreleri İle Hücre Zar1 Stabilitesi Yöntemine Göre Belirlenmesi (unpublished $\mathrm{Ph} . \mathrm{D}$. Thesis). Mustafa Kemal University Institute of Natural and Applied Sciences 251 p. 2010.

5. ALZAHRANI Y., KUŞVURAN A., ALHARBY H.F., KUŞVURAN S., RADY M.M. The defensive role of silicon in wheat against stress conditions induced by drought, salinity or cadmium. Ecotoxicology and Environmental Safety. 154, 187, 2018.

6. PATRIYAWATY N.R., RACHAPUTI RC., GEORGE D. Physiological mechanisms underpinning tolerance to high temperature stress during reproductive phase in mung bean (Vigna radiata (L.) Wilczek). Environmental and Experimental Botany, 150, 188, 2018.

7. RAI N., RAI K.K., TIWARI G., SINGH P.K. Changes in free radical generation, metabolites and antioxidant defense machinery in hyacinth bean (Lablab purpureus L.) in response to high temperature stress. Acta Physiologiae Plantarum 37 (3), 46, 2015.

8. CHAUKHANDE P., SHETRU A., HUNASHIKATTI L., BIRADAR G., SINGH B. Evaluation of French bean genotypes for high temperature tolerance using Temperature Induction Response (TIR) technique. Vegetable Science, 44 (2), 20, 2017.

9. HATFIELD J.L., PRUEGER J.H. Temperature extremes: effect on plant growth and development. Weather and Climate Extremes, 10, 41, 2015.

10. JUMRANI K., BHATIA V.S. Impact of combined stress of high temperature and water deficit on growth and seed yield of soybean. Physiology and Molecular Biology of Plants, 24 (1), 50, 2018

11. AKBAR A., SINGH MANOHAR S., TOTTEKKAAD VARIATH M., KURAPATI S., PASUPULETI J. Efficient partitioning of assimilates in stress-tolerant groundnut genotypes under high-temperature stress. Agronomy, 7 (2), 30, 2017.

12. QIAN Z. Relationship between Pepper Cultivars Yields and High Temperatures with Pepper in Different Genotypes. Agricultural Science Technology, 18 (4), 588, 2017.

13. KABAY T., ERDINÇ Ç., SENSOY S. Effects of drought stress on plant growth parameters membrane damage index and nutrient content in common bean genotypes. The Journal of Animal and Plant Sciences, 27 (3), 940, 2017.

14. BARIŞ A., ÇOBANOĞLU S., ÇAVUŞOĞLU Ş. Determination of changes in tastes of Ipsala and Kırkağaç melons against melon fly myiopardalis pardalina. Derim, 33 (1), 47. 2016.

15. KABAY T., SENSOY S. Enzyme, chlorophyll and ion changes in some common bean genotypes by high temperature stress. Ege University Journal of Agricultural Sciences, 54 (4), 429, 2017.

16. KABAY T. Effects of different Potassium Doses on growth and development of drought-sensitive bean plants. Acta Scientiarum Polonorum-Hortorum Cultus, 19, 62, 2020.
17. SANCHEZ-RODRIGUEZ E., RUBIO-WILHELMI M., CERVILLA L.M., BLASCO B., RIOS J.J., ROSALES M.A., RUIZ J.M. Genotypic differences in some physiological parameters symptomatic for oxidative stress under moderate drought in tomato plants. Plant Science, 178 (1), 30, 2010

18. ALP Y., KABAY T. The effect of drought stress on plant development in some native and commercial tomato genotypes. Yuzuncu Y1l University Journal of Agricultural Sciences. 27 (3), 387, 2017.

19. ZHOU R., KJÆR K.H., ROSENQVIST E., YU X., WU Z., OTTOSEN C.O. Physiological response to heat stress during seedling and anthesis stage in tomato genotypes differing in heat tolerance. Journal of Agronomy and Crop Science, 203 (1), 68, 2017.

20. KUŞVURAN, Ş. Relationships between physiological mechanisms for drought and salinity tolerance of melons (unpublished Ph.D. Thesis). Çukurova University Institute of Natural and Applied Sciences 356 p. 2010.

21. KOC S. Determination of Genotipic Variations for Salinity Tolerance at Early Plant Growth Stages in Green Beans. M.Sc. thesis, Institute of Natural and Applied Sciences University of Cukurova, 87, 2005.

22. BAĞCI E.G. Identification of drought-induced oxidative stress in chickpea with physiological and biochemical parameters (unpublished Ph.D. Thesis), Ankara University Faculty of Science. 403, 2010.

23. SANCHEZ F.J., ANDRES E.F., TENORIO J.L., AYERBE L. Growth of Epicotyls, Turgor Maintenance and Osmotic Adjustment in Pea Plants (Pisum sativum L.) Subjected to Water Stress. Field Crops Research, 86, 81, 2004.

24. TÜRKAN İ., BOR M., ÖZDEMIR F., KOCA H. Differential Responses of Lipid Peroxidation and Antioxidants in the Leaves of Drought-Tolerant P. acutifolius Gray and Drought Sensitive P. vulgaris L. Subjected to Polyethylene Glycol Mediates Water Stress. Plant Science, 168, 231, 2005.

25. DLUGOKECKA E., KACPERSKA-PALACZ A. ReExamination of Electrical Conductivity Method for Estimation of Drought Injury. Biologia Plantarum, 20, 262, 1978.

26. FAN S., BLAKE, T. Abscisic Acid Induced Electrolyte Leakage in Woody Species With Contrasting Ecological Requirements. Physiologia Plantarum, 90,419, 1994.

27. KABAY T., SENSOY S. Drought stress-induced changes in enzymes, chlorophyll and ions of some bean genotypes. Yuzuncu Yil University Journal of Agricultural Sciences, 26 (3), 380, 2016

28. YESILOVA A., DENIZHAN E. Modelling mite counts using Poisson and negative binomial. Fresenius Environmental Bulletin, 25, 5062, 2016.

29. KABAY T. Effects of Different Potassium Doses on Ion, Chlorophyll and Enzyme Contents of Drought Sensitive Bean Plants. Fresenius Environmental Bulletin, 27 (11): 7733, 2018.

30. KABAY T., ALP Y., SENSOY S. Effect of Vermicompost Application on Some Plant Characteristics in Lettuce (Lactuca Sativa L.). Fresenius Environmental Bulletin, 27 (12b), 9942, 2018a.

31. KABAY T., EKINCIALP A. ERDINÇ Ç., SENSOY S. The Impact of Low Temperatures on Plant Growth in Some Common Bean Genotypes. Fresenius Environmental Bulletin, 27 (12a), 8715, 2018b.

32. KABAY T. Effects of Different Potassium Doses on Development of High Temperature Sensitive Bean Plants. Fresenius Environmental Bulletin, 28 (1), 320, 2019. 